\title{
On 3d bulk geometry of Virasoro coadjoint orbits: orbit invariant charges and Virasoro hair on locally $\mathrm{AdS}_{3}$ geometries
}

\author{
M. M. Sheikh-Jabbari ${ }^{1, \mathrm{a}}$, H. Yavartanoo ${ }^{2, \mathrm{~b}}$ \\ ${ }^{1}$ School of Physics, Institute for Research in Fundamental Sciences (IPM), P.O.Box 19395-5531, Tehran, Iran \\ 2 State Key Laboratory of Theoretical Physics, Institute of Theoretical Physics, Chinese Academy of Sciences, Beijing 100190, China
}

Received: 29 June 2016 / Accepted: 18 August 2016 / Published online: 8 September 2016

(C) The Author(s) 2016. This article is published with open access at Springerlink.com

\begin{abstract}
Expanding upon [arXiv:1404.4472, arXiv:1511.06079], we provide a further detailed analysis of Bañados geometries, the most general solutions to the $\mathrm{AdS}_{3}$ Einstein gravity with Brown-Henneaux boundary conditions. We analyze in some detail the causal, horizon, and boundary structure, and the geodesic motion on these geometries, as well as the two classes of symplectic charges one can associate with these geometries: charges associated with the exact symmetries and the Virasoro charges. We elaborate on the one-to-one relation between the coadjoint orbits of two copies of the Virasoro group and Bañados geometries. We discuss that the information as regards the Bañados goemetries falls into two categories: "orbit invariant" information and "Virasoro hairs". The former concerns geometric quantities, while the latter are specified by the non-local surface integrals. We elaborate on multi-BTZ geometries which have a number of disconnected pieces at the horizon bifurcation curve. We study multi-BTZ black hole thermodynamics and discuss that the thermodynamic quantities are orbit invariants. We also comment on the implications of our analysis for a 2d CFT dual which could possibly be dual to $\mathrm{AdS}_{3}$ Einstein gravity.
\end{abstract}

\section{Contents}

1 Introduction $\ldots \ldots \ldots \ldots \ldots \ldots \ldots$

2 Bañados geometries and their causal structure . . . . 2

2.1 Diffeomorphisms preserving the Bañados gauge 3

2.2 Killing vectors of Bañados geometries . . . . . 3

2.3 Horizon and asymptotic boundary behavior . . 4

2.3.1 Killing horizons and bifurcation surfaces 4

2.3.2 More on causal and boundary structure . 6

2.4 Geodesic motion on generic Bañados geometries 10

\footnotetext{
a e-mail: jabbari@theory.ipm.ac.ir

be-mail: yavar@itp.ac.cn
}

2.5 Summary of Bañados geometries and outlook of the section . . . . . . . . . . 10

3 Bañados geometries and the associated conserved charges 11

3.1 Charges associated with symplectic non-exact symmetries (SNSs) . . . . . . . . . . . 11

3.2 Charges associated with symplectic exact symmetries (SESs) . . . . . . . . . . 11

3.3 Entropy, the first law and Smarr relation for Bañados geometries . . . . . . . . . . . 12

4 Virasoro coadjoint orbits and their associated geometries . 12

4.1 Constant representative coadjoint orbits . . . . 13

4.2 Nonconstant representative coadjoint orbits . . 14

4.3 Orbit invariant quantities . . . . . . . . . . 15

4.4 Bañados geometries/Virasoro orbits correspondence ................. 15

5 Concluding remarks and outlook . . . . . . . . 16

Appendix A: More on constant representative orbits and their geometries . . . . . . . . . . . 17

A.1: More on geometry of constant $L_{ \pm}$cases $\ldots .17$

A.2: Geodesic motion on massless BTZ . . . . . . 18

Null and time-like geodesies of BTZ black holes . . 19

A.3: More on geometries of constant representative orbits . . . . . . . . . . 20 20

References . . . . . . . . . . . . . . 2 21

\section{Introduction}

Three-dimensional gravity has very interesting features and has been long viewed as a testing ground for ideas in semiclassical and quantum gravity [1-4]. 3d Einstein gravity on flat (or $\mathrm{AdS}_{3}, \mathrm{dS}_{3}$ ) backgrounds does not have propagating degrees of freedom and all solutions to these theories are locally flat (or $\mathrm{AdS}_{3}, \mathrm{dS}_{3}$ ). Nevertheless, it is well known that it admits nontrivial solutions [5,6], including black holes $[7,8]$. One can in fact classify all the solutions to this theory with prescribed boundary conditions. In this paper we will focus on the $\mathrm{AdS}_{3}$ gravity case and analyze a family of 
locally $\mathrm{AdS}_{3}$ geometries with Brown-Henneaux boundary conditions [9], namely with flat $2 \mathrm{~d}$ metric at the boundary. ${ }^{1}$

These solutions, the Bañados geometries, which were first discussed in [11], are specified by two arbitrary periodic functions $L_{+}=L_{+}\left(x^{+}\right)$and $L_{-}=L_{-}\left(x^{-}\right), L_{ \pm}\left(x^{ \pm}+2 \pi\right)=$ $L_{ \pm}\left(x^{ \pm}\right)$. Although all Bañados geometries are locally $\mathrm{AdS}_{3}$ and locally diffeomorphic to each other, it is now established that these geometries are physically distinct, as one can specify them with quasi-local, conserved surface charges; see [12] and the references therein for a recent detailed study. The latter feature is also reflected in the fact that there are no everywhere smooth coordinate transformations which respect the periodicity in $x^{ \pm}$and can render these geometries global or Poincaré patches $\mathrm{AdS}_{3}$ [12] (see also [13-15]).

In fact, one can distinguish two kinds of such conserved charges: those associated with exact symmetries (Killing symmetries) of the Bañados solutions and those which are in the family of symplectic symmetries. ${ }^{2}$ See $[12,16]$ for a detailed discussion on the concepts and the terminology.

If we denote the generators of the exact symmetries by $J_{ \pm}$and those of symplectic symmetries by $L_{n}, \bar{L}_{n}$, one can show that the latter form two (left and right) copies of Virasoro algebras at Brown-Henneaux central charge $c$ [9] and that $J_{ \pm}$commute with each other and also with all $L_{n}, \bar{L}_{n}$ [12]. Since these two sets are commuting, one may hence label the geometries by $J_{ \pm}$as well as the Virasoro charges. As discussed in [12] (see also [14,15,17,18]) one may then classify Bañados geometries by the product of the two, left and right, Virasoro coadjoint orbits [19,20]. Each Virasoro coadjoint orbit which is in correspondence with a "representation" (an orbit) of the Virasoro group, is generically labeled by an integer and a continuous real number ${ }^{3}$ and then "states" in a given orbit are fully specified once we also give their Virasoro charges, the "Virasoro hairs". The goal of this paper is to elaborate on the results of $[12,21]$ and on the one-to-one relation between two copies of Virasoro orbits and Bañados geometries.

The picture we depict here will correct and complete the one given in [21]: The information as regards Bañados geometries available to local observables of the usual classical GR, the geometric notions such as geodesic length, and causal and boundary structure, and the black hole (thermo)dynamics quantities like surface gravity and horizon angular velocity, entropy, concerns "orbit invariant quanti-

\footnotetext{
1 More general solutions are also possible on relaxing these boundary conditions [10].

2 Note that the notion of symplectic symmetries extends the notion of asymptotic symmetries discussed in [9], in the sense that the charges are not only defined at the $\mathrm{AdS}_{3}$ boundary, but also on any codimension two, compact, space-like curve in the bulk.

3 As we will discuss in detail in Sect. 4, these two labels are not enough to uniquely specify the orbit and we need to also specify the type of the orbit.
}

ties". That is, all geometries which fall into the same orbit share these properties, regardless of their "Virasoro hair". On the other hand, the information as regards the Virasoro hair is semiclassical, in the sense that it is of the form of surface non-local ("quasi-local") charges; the Virasoro charges may be viewed as the "hair" on classical geometries which all have the same mass, angular momentum, and causal structure. Given this picture, one may hope to obtain a full quantum description upon quantization of Virasoro coadjoint orbits. We shall provide some discussion of the latter point in the end; a thorough analysis is left to upcoming work.

To this end, in Sect. 2 we analyze geometric aspects of Bañados solutions. This includes reviewing their Killing vectors [21], analyzing the horizon, causal and boundary structure of these geometries and the geodesic motion on these geometries. In Sect. 3, we analyze charges associated with Bañados metrics. In Sect. 4, after reviewing Virasoro coadjoint orbits and their classification, we discuss the $3 \mathrm{~d}$ Bañados geometry associated with Virasoro coadjoint orbits. The last section is devoted to a summary and outlook. In an appendix we have given detailed analysis of special, but important cases, the geometries corresponding to Virasoro coadjoint orbits with constant character representative.

\section{Bañados geometries and their causal structure}

In this paper we focus on the most general solutions to the $\mathrm{AdS}_{3}$ Einstein gravity equations,

$R_{\mu \nu}=-\frac{2}{\ell^{2}} g_{\mu \nu}$

with Brown-Henneaux boundary conditions [9]. These solutions are all locally $\mathrm{AdS}_{3}$ with local $\operatorname{sl}(2, \mathbb{R}) \times \operatorname{sl}(2, \mathbb{R})$ isometries and their causal boundary is a $2 \mathrm{~d}$ cylinder (in orthonormal coordinates). One may relax the behavior at the boundary and get more general class of solutions [10,22]. The Bañados solutions in the Fefferman-Graham gauge [23] is given by [11]

$\mathrm{d} s^{2}=\ell^{2} \frac{\mathrm{d} r^{2}}{r^{2}}-\left(r \mathrm{~d} x^{+}-\frac{\ell^{2}}{r} L_{-} \mathrm{d} x^{-}\right)\left(r \mathrm{~d} x^{-}-\frac{\ell^{2}}{r} L_{+} \mathrm{d} x^{+}\right)$,

where $L_{+}=L_{+}\left(x^{+}\right)$and $L_{-}=L_{-}\left(x^{-}\right)$are arbitrary, smooth, periodic functions, $L_{ \pm}\left(x^{ \pm}+2 \pi\right)=L_{ \pm}\left(x^{ \pm}\right)$. We assume here that $x^{ \pm} \in[0,2 \pi]$ and parametrize two circles. The $x^{ \pm}, r$ coordinate system used in (2.2) will be called a Bañados coordinate system or a Bañados gauge. A similar solution in other gauges, e.g. in the Gaussian null coordinates (also called BMS gauge) has been constructed and analyzed $[12,24]$. For the metrics $(2.2)$,

$\operatorname{det} g=-\frac{\ell^{2}}{4 r^{6}}\left(r^{4}-r_{0}^{4}\right)^{2}, \quad r_{0}^{4} \equiv \ell^{4} L_{+} L_{-}$. 
On the range of the $r$ coordinate The metric (2.2) may be written in terms of $r^{2}$ (and not just $r$ ). Therefore, in principle, $r^{2}$ can assume positive or negative real values. However, at large $\left|r^{2}\right|$, the metrics (2.2) take the form

$\mathrm{d} s^{2} \approx \ell^{2} \frac{\mathrm{d} r^{2}}{r^{2}}-r^{2} \mathrm{~d} x^{+} \mathrm{d} x^{-}$

and without loss of generality one may choose $x^{ \pm}=\tau \pm$ $\varphi$, where $\varphi \in[0,2 \pi]$ is a space-like circle, while $\tau$ is a time-like coordinate. This is in accord with the fact that the causal boundary of the geometry is (part of) the cylinder parametrized by $x^{ \pm}$. With this choice, to avoid the appearance of closed time-like curves (CTCs), $\left|r^{2}\right|$ cannot take a large negative value. We shall cut the $r^{2}$ range from a negative value, $r_{\mathrm{CTC} 1}^{2}$, where CTC develops and we take $r_{\mathrm{CTC} 1}^{2}<$ $r^{2}$. This range of $r^{2}$, however, is not necessarily CTC free. As we will argue in the next section, there is still a range $r_{\mathrm{CTC} 2}^{2}<r^{2}<0$, with $r_{\mathrm{CTC} 1}^{2} \leq r_{\mathrm{CTC} 2}^{2}<0$, where we have CTCs. We should cut out this range too. The acceptable range of $r^{2}$ will then contain two disconnected pieces: $r_{\mathrm{CTC} 1}^{2} \leq$ $r^{2} \leq r_{\mathrm{CTC} 2}^{2}<0$ and $r^{2}>0$. Moreover, as we will argue this allowed range of $r^{2}$ covers the geometry twice. As a rough argument for the latter, note that if $r_{0}^{4}>0$ then in the $r^{2} \ll 1$ region the metric essentially takes the same form as $r^{2} \gg 1$ region. This will become more clear in the level Penrose diagrams shown in the next section. For illustrative purposes we discuss the special case of a BTZ black hole $[7,8]$, corresponding to constant positive $L_{ \pm}$in the appendix and compare the relation between Bañados and the more standard BTZ-coordinate systems.

\subsection{Diffeomorphisms preserving the Bañados gauge}

The geometries (2.2) are written in a specific coordinate system, the Bañados gauge. This coordinate system extends the Fefferman-Graham coordinates (which are usually defined near the boundary at large $r$ ), to arbitrary $r$. The Bañados gauge is hence defined by the "gauge fixing conditions"

$g_{r r}=\frac{1}{r^{2}}, \quad g_{r+}=g_{r-}=0$.

One may then ask what the "residual" diffeomorphisms are [25] which preserve the Bañados gauge. This question was explored and answered in [12]:

$$
\begin{aligned}
& \chi\left[\epsilon_{+}, \epsilon_{-}\right]=\chi^{r} \partial_{r}+\chi^{+} \partial_{+}+\chi^{-} \partial_{-}, \\
& \chi^{r}=-\frac{r}{2}\left(\epsilon_{+}^{\prime}+\epsilon_{-}^{\prime}\right), \quad \chi^{+}=\epsilon_{+}+\frac{\ell^{2} r^{2} \epsilon_{-}^{\prime \prime}+\ell^{4} L_{-} \epsilon_{+}^{\prime \prime}}{2\left(r^{4}-\ell^{4} L_{+} L_{-}\right)}, \\
& \chi^{-}=\epsilon_{-}+\frac{\ell^{2} r^{2} \epsilon_{+}^{\prime \prime}+\ell^{4} L_{+} \epsilon_{-}^{\prime \prime}}{2\left(r^{4}-\ell^{4} L_{+} L_{-}\right)}
\end{aligned}
$$

where $\epsilon_{ \pm}=\epsilon_{ \pm}\left(x^{ \pm}\right)$are two arbitrary, periodic functions, i.e. $\epsilon_{ \pm}\left(x^{ \pm}\right)=\epsilon_{ \pm}\left(x^{ \pm}+2 \pi\right)$, and the prime denotes a derivative with respect to the argument.

Although $\chi$ diffeomorphisms keep the form of the metric invariant, they generically shift the functions $L_{ \pm}$. From the Lie derivative of the metric $\mathcal{L}_{\chi} g_{\mu \nu}$ one can read off [12]

$\delta_{\chi} L_{+}=-\frac{1}{2} \epsilon_{+}^{\prime \prime \prime}+\epsilon_{+} L_{+}^{\prime}+2 \epsilon_{+}^{\prime} L_{+}$,
$\delta_{\chi} L_{-}=-\frac{1}{2} \epsilon_{-}^{\prime \prime \prime}+\epsilon_{-} L_{-}^{\prime}+2 \epsilon_{-}^{\prime} L_{-}$.

\subsection{Killing vectors of Bañados geometries}

Killing vectors $\zeta$, with $\mathcal{L}_{\zeta} g=0$, should have the form (2.5) but for specific $\epsilon$ 's satisfying $\delta_{\zeta} L_{ \pm}=0$. We will denote the corresponding $\epsilon$ 's by $K_{ \pm}$. Therefore, the Killing vectors are of the form [21]

$\zeta\left[K_{+}, K_{-}\right]=\zeta^{r} \partial_{r}+\zeta^{+} \partial_{+}+\zeta^{-} \partial_{-}$,

$\zeta^{r}=-\frac{r}{2}\left(K_{+}^{\prime}+K_{-}^{\prime}\right), \quad \zeta^{+}=K_{+}+\frac{\ell^{2} r^{2} K_{-}^{\prime \prime}+\ell^{4} L_{-} K_{+}^{\prime \prime}}{2\left(r^{4}-\ell^{4} L_{+} L_{-}\right)}$,

$\zeta^{-}=K_{-}+\frac{\ell^{2} r^{2} K_{+}^{\prime \prime}+\ell^{4} L_{+} K_{-}^{\prime \prime}}{2\left(r^{4}-\ell^{4} L_{+} L_{-}\right)}$,

where now $K_{ \pm}$should satisfy the following equations:

$K_{+}^{\prime \prime \prime}-4 K_{+}^{\prime} L_{+}-2 K_{+} L_{+}^{\prime}=0$,

$K_{-}^{\prime \prime \prime}-4 K_{-}^{\prime} L_{-}-2 K_{-} L_{-}^{\prime}=0$.

Since the notion of a Killing vector is a local one, all solutions to (2.8) generate a Killing vector, regardless of the fact that the corresponding $K_{ \pm}$are periodic or not. The above third order equations have six linearly independent solutions. These solutions constitute the six local isometries of Bañados geometries which satisfy the $\operatorname{sl}(2, \mathbb{R}) \times \operatorname{sl}(2, \mathbb{R})$ algebra, as expected, recalling that Bañados geometries are locally $\mathrm{AdS}_{3}$ [21]. The $K_{ \pm}\left(x^{ \pm}\right)$functions which solve (2.8) are not necessarily periodic. Therefore, not all of the associated six Killing vector field are "globally defined".

It was noted in [20,21] that (2.8) may be solved through a second order Schrödinger type equation:

$\psi^{\prime \prime}-L_{+} \psi=0, \quad \phi^{\prime \prime}-L_{-} \phi=0$.

If the two linearly independent solutions to the above equations are denoted $\psi_{1}, \psi_{2}$, and $\phi_{1}, \phi_{2}$, then it is easy to verify that

$K_{+}^{-} \equiv \frac{1}{2} \psi_{1}^{2}, \quad K_{+}^{+} \equiv \frac{1}{2} \psi_{2}^{2}, \quad K_{+}^{0} \equiv \frac{1}{2} \psi_{1} \psi_{2}$,

$K_{-}^{+} \equiv \frac{1}{2} \phi_{1}^{2}, \quad K_{-}^{+} \equiv \frac{1}{2} \phi_{2}^{2}, \quad K_{-}^{0} \equiv \frac{1}{2} \phi_{1} \phi_{2}$

provide the three linearly independent solutions to (2.8) where we adopt the normalization 
$\psi_{1}^{\prime} \psi_{2}-\psi_{1} \psi_{2}^{\prime}=1, \quad \phi_{1}^{\prime} \phi_{2}-\phi_{1} \phi_{2}^{\prime}=1$.

Recalling that $L_{ \pm}$are periodic smooth functions, the Floquet theorem implies that $[21,26]$

$\psi_{1}=e^{\mathcal{T}_{+} x^{+}} P_{1}\left(x^{+}\right), \quad \psi_{2}=e^{-\mathcal{T}_{+} x^{+}} P_{2}\left(x^{+}\right)$,

$\phi_{1}=e^{\mathcal{T}_{-} x^{-}} Q_{1}\left(x^{-}\right), \quad \phi_{2}=e^{-\mathcal{T}_{-} x^{-}} Q_{2}\left(x^{-}\right)$,

where $P_{i}, Q_{i}$ are periodic smooth functions and $\mathcal{T}_{ \pm}$are two constants built from $L_{ \pm}$. In general $\mathcal{T}_{ \pm}$are two complex numbers and without loss of generality we can take $\operatorname{Re}\left(\mathcal{T}_{ \pm}\right) \geq 0$. The discussion of [26] reveals that, although $P_{i}, Q_{i}$ are generically $4 \pi$ periodic, $P_{1} P_{2}$ and $Q_{1} Q_{2}$, and hence combinations like $\psi_{1} \psi_{2}, \psi_{1} / \psi_{2}, \psi_{1}^{\prime} / \psi_{1}$, and $\psi_{2}^{\prime} / \psi_{2}$, which have geometric meaning, are all $2 \pi$ periodic. Therefore, in the general case with $\operatorname{Re}\left(\mathcal{T}_{ \pm}\right) \neq 0$, only two of the six Killing vectors, those generated by $K_{+}^{0}, K_{-}^{0}$, are periodic. Hereafter, we will use the notation

$K_{+}=\psi_{1} \psi_{2}, \quad K_{-}=\phi_{1} \phi_{2}$,

for the functions generating the two periodic global Killing vectors, which will be denoted by $\zeta_{+}$and $\zeta_{-}$. In other words, the Bañados geometriesin general have $U(1)_{+} \times U(1)_{-}$compact isometries. ${ }^{4}$

The above construction makes it clear that solutions to (2.9), and hence solutions to (2.8), are implicit functions of $L_{ \pm}$, i.e. $K_{+}=K_{+}\left(L_{+}\left(x^{+}\right)\right), K_{-}=K_{-}\left(L_{-}\left(x^{-}\right)\right)$. In particular, we note that $K_{ \pm}$are only functions of $L_{ \pm}$and not their derivatives $L_{ \pm}^{\prime}$.

\subsection{Horizon and asymptotic boundary behavior}

Any linear combination of two global Killing vector fields $\zeta_{ \pm}$is also a Killing vector. One may easily check that with the normalization (2.10) and (2.12), $\left|\zeta_{+}\right|^{2}=\left|\zeta_{-}\right|^{2}=\ell^{2} / 4$, while $\zeta_{+} \cdot \zeta_{-} \neq 0$ is a nontrivial function. There is a certain combination of the two Killing vectors which are normal to each other:

$\zeta_{\mathcal{H}_{ \pm}} \equiv \zeta_{+} \pm \zeta_{-}$.

The norm of these vectors is given as

$\left|\zeta_{\mathcal{H}_{ \pm}}\right|^{2}=\mp \frac{\psi_{1} \psi_{2} \phi_{1} \phi_{2}}{r^{2}}\left(r^{2}-r_{1 \pm}^{2}\right)\left(r^{2}-r_{2 \pm}^{2}\right)$,

where

$r_{1+}^{2}=\ell^{2} \frac{\psi_{1}^{\prime} \phi_{1}^{\prime}}{\psi_{1} \phi_{1}}, \quad r_{2+}^{2}=\ell^{2} \frac{\psi_{2}^{\prime} \phi_{2}^{\prime}}{\psi_{2} \phi_{2}}, \quad r_{1-}^{2}=\ell^{2} \frac{\psi_{1}^{\prime} \phi_{2}^{\prime}}{\psi_{1} \phi_{2}}$,

$r_{2-}^{2}=\ell^{2} \frac{\psi_{2}^{\prime} \phi_{1}^{\prime}}{\psi_{2} \phi_{1}}$

\footnotetext{
${ }^{4}$ There are special $\operatorname{Re}\left(\mathcal{T}_{+}\right)$and/or $\operatorname{Re}\left(\mathcal{T}_{-}\right)=0$ cases where more Killing vectors are periodic. These cases may have four or six global Killing vectors. The latter happens only for global $\mathrm{AdS}_{3}$ with $L_{ \pm}=$ $-1 / 4, \mathcal{T}_{ \pm}=0$ where we have an $\operatorname{Sl}(2, \mathbb{R}) \times \operatorname{Sl}(2, \mathbb{R})$ global isometry.
}

For later use we note that $r_{1+}^{2} r_{2+}^{2}=r_{1-}^{2} r_{2-}^{2}$ and

$$
\begin{aligned}
& r_{1+}^{2}-r_{1-}^{2}=\frac{\ell^{2}}{\phi_{1} \phi_{2}} \frac{\psi_{1}^{\prime}}{\psi_{1}}, \quad r_{2+}^{2}-r_{2-}^{2}=-\frac{\ell^{2}}{\phi_{1} \phi_{2}} \frac{\psi_{2}^{\prime}}{\psi_{2}}, \\
& r_{1+}^{2}-r_{2-}^{2}=\frac{\ell^{2}}{\psi_{1} \psi_{2}} \frac{\phi_{1}^{\prime}}{\phi_{1}}, \quad r_{2+}^{2}-r_{1-}^{2}=-\frac{\ell^{2}}{\psi_{1} \psi_{2}} \frac{\phi_{2}^{\prime}}{\phi_{2}} .
\end{aligned}
$$

Using these identities one may readily check that

$\zeta_{\mathcal{H}_{+}} \cdot \zeta_{\mathcal{H}_{-}}=0,\left.\quad\left|\zeta_{\mathcal{H}_{ \pm}}\right|^{2}\right|_{\left|\zeta_{\mathcal{H}_{\mp}}\right|^{2}=0}=\ell^{2}$

Note Although most of the statements in this subsection are also true for generic $\mathcal{T}_{ \pm}$[cf. (2.11)], in Sects. 2 and 3, we will be assuming that $\mathcal{T}_{ \pm}$are real-valued.

\subsubsection{Killing horizons and bifurcation surfaces}

At the surfaces where the Killing vectors $\zeta_{\mathcal{H}_{+}}$become null we have Killing horizons. Explicitly, either of the four codimension one surfaces $\mathcal{H}_{\alpha \sigma}$,

$\mathcal{H}_{\alpha \sigma}: \quad r^{2}=r_{\alpha \sigma}^{2}, \quad \alpha=1,2, \sigma=+,-$,

defines a null surface along the corresponding null Killing vector.

We will show below that:

- If $r_{\alpha \sigma}^{2}$ is in the acceptable range [where geometry is CTC free, cf. the discussion in the paragraph below (2.3)], then we have Killing horizons.

- These horizons are generically (not always) bifurcateKilling horizons. We specify what is the bifurcation curve.

- When they exist, these Killing horizons are either event horizon (outer horizon) or Cauchy horizon (inner horizon); if the event horizon is generated by $\zeta_{\mathcal{H}_{ \pm}}$, the inner horizon is generated by $\zeta_{\mathcal{H}_{\mp}}$.

- Then $\mathcal{H}_{\alpha \pm}$ null surfaces for $\alpha=1,2$ correspond to two branches of the event horizon and vice versa, and the inner and outer bifurcation curves are $\mathcal{H}_{1 \pm} \cap \mathcal{H}_{2 \pm}$, and they are given by the equation $r^{2}=r_{1 \pm}^{2}=r_{2 \pm}^{2}$.

To ensure the existence of horizons and whether they are inner or outer horizons, we need to analyze the signs, zeros, and infinities of $r_{\alpha \pm}^{2}$ and $\left|\zeta_{\mathcal{H}_{ \pm}}\right|^{2}$. As discussed, the range of $r$ coordinate can contain $r^{2}<0$ regions. The CTC appears in the regions where both of $\zeta_{\mathcal{H}_{ \pm}}$are spacelike. If the condition for the existence of horizons cannot be met in all of the $x^{ \pm} \in[0,2 \pi]$ region, we are forced to cut some parts (due to CTC). Moreover, since in general 
$\psi$ and $\phi$ have zeros, the horizon and boundary regions are not simply connected and will generically have some disconnected patches. Another point we will discuss below is that the allowed range for $r^{2}$ gives a double cover of spacetime. The latter fact, that the $r^{2}<0$ should also be included in the coordinate range and the existence of CTC's, may be seen very explicitly for the BTZ case of constant, positive $L_{ \pm}$, which is discussed in Appendix A.1. The analysis of the causal structure will be quite different for constant and the nonconstant cases and they need to be discussed separately.

Case I: constant $L_{ \pm}$This case is the more studied and better understood case; see, e.g., $[12,21]$ and the references therein. For the case where $L_{+} \geq 0$ we have BTZ black holes. This case will be discussed in some detail in the appendix. For the BTZ case, as is well known [7,8], we generically have a (bifurcate) simply connected horizon which is a circle.

Case II: generic $L_{ \pm}$case Unlike Case I, in this case $\psi$ and $\phi$ functions can have roots and zeros. Here and below, unless mentioned explicitly, we will be considering nonconstant generic $L_{ \pm}$cases. Before starting the analysis, we note the facts that, as implied by (2.9) and (2.10):

- $\psi_{1}, \psi_{2}$ are smooth functions and can only have simple roots.

- $\psi_{1}^{\prime}, \psi_{2}^{\prime}$ are also smooth and can only have simple roots.

- The number of simple roots of $\psi_{1}$ in $x^{+} \in[0,2 \pi]$ is equal to the number of roots of $\psi_{2}$ in the same range. Let us denote this number by $n_{+}$. One may show that $\psi_{1}^{\prime}, \psi_{2}^{\prime}$ have the same number of roots [26].

- Between any two roots of $\psi_{1}\left(\psi_{2}\right)$, there is a root of $\psi_{2}$ $\left(\psi_{1}\right)$, and similarly for their derivatives [26].

- If the roots of $\psi_{2}, \psi_{1}^{\prime}, \psi_{2}^{\prime}, \psi_{1}$ are, respectively, denoted by $x_{1, i}^{+}, x_{2, i}^{+}, x_{3, i}^{+}, x_{4, i}^{+}, i=1, \ldots, n_{+}$, we have $x_{1, i}^{+}<$ $x_{2, i}^{+}<x_{3, i}^{+}<x_{4, i}^{+}$and $x_{4, i}^{+}<x_{1, i+1}^{+}$. Note that we are using the normalization (2.10).

- Considering the roots, we can divide the $[0,2 \pi]$ range into $4 n_{+}$regions:

$$
\begin{aligned}
I_{1,1} & =\left[0, x_{1,1}^{+}\right), I_{2,1}=\left(x_{1,1}^{+}, x_{2,1}^{+}\right), \\
I_{3,1} & =\left(x_{2,1}^{+}, x_{3,1}^{+}\right), I_{4,1}=\left(x_{3,1}^{+}, x_{4,1}^{+}\right), \\
I_{1,2} & =\left(x_{4,1}^{+}, x_{1,2}^{+}\right), I_{2,2}=\left(x_{1,2}^{+}, x_{2,2}^{+}\right), \ldots, \\
I_{3, n_{+}} & =\left(x_{2, n_{+}}^{+}, x_{3, n_{+}}^{+}\right), I_{4, n_{+}}=\left(x_{3, n_{+}}^{+}, x_{4, n_{+}}^{+}\right) .
\end{aligned}
$$

The range $\left(x_{4, n_{+}}^{+}, 2 \pi\right]$ is identified with the $I_{1,1}$ region.

- Let us focus on the $i$ th roots, i.e. the $I_{a, i}, a=1,2,3,4$ regions. One can always choose the overall sign of $\psi_{1}$ and $\psi_{2}$ functions such that

\begin{tabular}{llllll}
\hline Function & \multicolumn{2}{l}{ Region } \\
\cline { 2 - 5 } & $I_{1, i}$ & $I_{2, i}$ & $I_{3, i}$ & $I_{4, i}$ & $I_{1, i+1}$ \\
\hline$\psi_{1}$ & + & + & + & + & - \\
$\psi_{2}$ & + & - & - & - & - \\
$\psi_{1}^{\prime}$ & + & + & - & - & - \\
$\psi_{2}^{\prime}$ & - & - & - & + & + \\
$\psi_{1} \psi_{2}$ & + & - & - & - & + \\
$\psi_{1}^{\prime} / \psi_{1}$ & + & + & - & - & + \\
$\psi_{2}^{\prime} / \psi_{2}$ & - & + & + & - & - \\
\hline
\end{tabular}

Note that for $2 \pi$ periodic functions like $\psi_{1} \psi_{2}$ and $\psi_{\alpha}^{\prime} / \psi_{\alpha}$, the first and fifth column are the same.

- In a similar fashion the $\phi$ functions may have $n_{-}$roots, with the same properties and ordering.

On the existence of horizons The horizons, if they exist, should be at the roots of $\left|\zeta_{\mathcal{H}_{ \pm}}\right|^{2}$. To distinguish which one is the inner horizon and which one the outer, we need to study the sign of $r_{\alpha \pm}^{2}$ functions. Given the above analysis on the roots and signs of $\psi, \phi$ 's, we learn that $r_{1 \pm}^{2}, r_{2 \pm}^{2}$ in the $I_{a, i}^{+}$ and $I_{b, j}^{-}$regions, have the signs given in Table 1 .

The event horizon The outer (event) horizon is by definition the null surface which is the boundary of all the past or future light-cones of points at the $\mathrm{AdS}_{3}$ boundary. For the $\mathrm{AdS}_{3}$ case, the Killing vector field generating the event horizon remains time-like at the boundary, ${ }^{5}$ while becoming null at the horizon. In order to distinguish which of $r_{\alpha+}$ or $r_{\alpha-}$ gives the outer (event) horizons we need to distinguish which of $\zeta_{\mathcal{H}_{ \pm}}$are time-like at the boundary (large $r^{2}$ region). From (2.14) we learn that for large $r^{2}$

$\left|\zeta_{\mathcal{H}_{ \pm}}\right|^{2} \approx \mp r^{2} \Pi, \quad \Pi \equiv \phi_{1} \phi_{2} \psi_{1} \psi_{2}$,

and the sign of $\Pi$ in different regions is given in Table 2 .

Inner and outer horizons and horizon radius differences The criterion above will distinguish which of the $r_{\alpha+}^{2}$ or $r_{\alpha-}^{2}$ correspond to bifurcate event horizon(s). One may distinguish which is the inner (Cauchy) horizon noting the following: The outer horizon is by definition the one which is closer to the boundary than the inner horizon. That is, the outer horizon should occur at a larger radius than the inner horizon. The signs of horizon radius differences are given in Table 3.

How to build full $4 n_{+} \times 4 n_{-}$tables We note that, as discussed above, if in general the functions $\psi$ and $\phi$ have, respectively, $n_{+}$and $n_{-}$zeros, then the above tables, instead of being $5 \times 5$ should have $4 n_{+} \times 4 n_{-}=16 n_{+} n_{-}$regions. The way to build

\footnotetext{
5 We note that this is a generic property of $\mathrm{AdS}_{3}$ black holes and is unlike the asymptotic flat black holes like the Kerr black hole. In the Kerr case the horizon generating Killing vector is generically spacelike in the asymptotic region of the spacetime, and it becomes time-like only in a region very close to the axis of rotation (usually denoted by $\theta=0, \pi)$.
} 
Table 1 Left and right top tables, respectively, show sign of $r_{1+}^{2}$ and $r_{2+}^{2}$. Left and right bottom tables, respectively, show the signs of $r_{1-}^{2}$ and $r_{2-}^{2}$. Note that the change of sign for either of these functions happens

\begin{tabular}{|c|c|c|c|c|c|}
\hline$x^{+} x^{-}$ & $I_{1, j}^{-}$ & $I_{2, j}^{-}$ & $I_{3, j}^{-}$ & $I_{4, j}^{-}$ & $I_{1, j+1}^{-}$ \\
\hline$I_{1, i}^{+}$ & + & + & - & - & + \\
\hline$I_{2, i}^{+}$ & + & + & - & - & + \\
\hline$I_{3, i}^{+}$ & - & - & + & + & - \\
\hline$I_{4, i}^{+}$ & - & - & + & + & - \\
\hline$I_{1, i+1}^{+}$ & + & + & - & - & + \\
\hline \hline$x^{-}$ & $I_{1, j}^{-}$ & $I_{2, j}^{-}$ & $I_{3, j}^{-}$ & $I_{4, j}^{-}$ & $I_{1, j+1}^{-}$ \\
\hline$x^{+}$ & - & + & + & - & - \\
\hline$I_{1, i}^{+}$ & - & + & + & - & - \\
\hline$I_{2, i}^{+}$ & - & - & - & + & + \\
\hline$I_{3, i}^{+}$ & + & - & - & + & + \\
\hline$I_{4, i}^{+}$ & + & - & - & + \\
\hline$I_{1, i+1}^{+}$ & - & + & + & - & - \\
\hline \multicolumn{2}{r}{} \\
\hline
\end{tabular}

Table 2 Sign of $\Pi$. Sign of norm of $\left|\zeta_{\mathcal{H}_{ \pm}}\right|^{2}$ near the boundary is $\mp \Pi$

\begin{tabular}{llllll}
\hline$x^{+}$ & $x^{-}$ & & & & \\
\cline { 2 - 6 } & $I_{1, j}^{-}$ & $I_{2, j}^{-}$ & $I_{3, j}^{-}$ & $I_{4, j}^{-}$ & $I_{1, j+1}^{-}$ \\
\hline$I_{1, i}^{+}$ & + & - & - & - & + \\
$I_{2, i}^{+}$ & - & + & + & + & - \\
$I_{3, i}^{+}$ & - & + & + & + & - \\
$I_{4, i}^{+}$ & - & + & + & + & - \\
$I_{1, i+1}^{+}$ & + & - & - & - & + \\
\hline
\end{tabular}

these bigger tables from those given here is simply copying the above tables $n_{-}$times on the rows, identifying the last and first columns in each copy; and copying $n_{+}$times the columns and then identifying the last and first rows in each copy. ${ }^{6}$ In the end, we should also identify the first row and the last rows, and the first column and the last column. This last identification is to implement the $2 \pi$ periodicity of the $\Pi$ and $r_{\alpha \pm}^{2}$ functions.

We also note that the tables here denote the cylinder spanned by $x^{ \pm}$with $x^{ \pm} \in[0,2 \pi]$. These tables are hence, in fact, depictions of the $\mathrm{AdS}_{3}$ boundary.

\subsubsection{More on causal and boundary structure}

Equipped with the information of the signs of $\left|\zeta_{\mathcal{H}_{ \pm}}\right|^{2}$ and $r_{\alpha \pm}^{2}$, we are now ready to build the full causal structure of

\footnotetext{
${ }^{6}$ As we can explicitly see, in our $5 \times 5$ tables the first and fifth rows and columns are the same.
}

at places where they vanish, or when they become infinite. The former happens at zeros of derivatives $\psi^{\prime}, \phi^{\prime}$, while the latter occurs at zeros of $\psi$ and $\phi$

\begin{tabular}{|c|c|c|c|c|c|}
\hline$x^{+} x^{-}$ & $I_{1, j}^{-}$ & $I_{2, j}^{-}$ & $I_{3, j}^{-}$ & $I_{4, j}^{-}$ & $I_{1, j+1}^{-}$ \\
\hline$I_{1, i}^{+}$ & + & - & - & + & + \\
\hline$I_{2, i}^{+}$ & - & + & + & - & - \\
\hline$I_{3, i}^{+}$ & - & + & + & - & - \\
\hline$I_{4, i}^{+}$ & + & - & - & + & + \\
\hline$I_{1, i+1}^{+}$ & + & - & - & + & + \\
\hline$x^{-}$ & $I_{1, j}^{-}$ & $I_{2, j}^{-}$ & $I_{3, j}^{-}$ & $I_{4, j}^{-}$ & $I_{1, j+1}^{-}$ \\
\hline$x^{+}$ & - & - & + & + & - \\
\hline$I_{1, i}^{+}$ & - & + & - & - & + \\
\hline$I_{2, i}^{+}$ & + & + & + & - \\
\hline$I_{3, i}^{+}$ & + & + & - & - & + \\
\hline$I_{4, i}^{+}$ & - & - & + & + & - \\
\hline$I_{1, i+1}^{+}$ & - & - & + & + & - \\
\hline
\end{tabular}

the Bañados geometries and discuss horizon properties like geometry of inner and bifurcation curves, and the horizon angular velocity and surface gravity. As we will discuss, the geometry, the boundary, and the event horizon in general consists of some number of causally disconnected pieces. To gain a better intuition and picture, however, we would like to invite the reader to go through Appendix A.1 where we discuss the simpler case of constant $L_{ \pm}$.

Surface gravity One may show

$-\left.\frac{1}{4}\left|\nabla \zeta_{\mathcal{H}_{ \pm}}\right|^{2}\right|_{\left|\zeta_{\mathcal{H}_{ \pm}}\right|^{2}=0}=1$

implying that the "un-normalized surface gravity" at the Killing horizons are equal (up to a sign). We stress that this equation is true for all four choices of roots of $\left|\zeta_{\mathcal{H}_{+}}\right|$. To read the physical surface gravity and determining its sign, however, we need to fix the normalization of the Killing vectors.

Fixing the normalization of Killing vectors It is well known that in order to read off the horizon's kinematical properties, like the horizon angular velocity and surface gravity, one needs to choose an appropriate normalization for the corresponding Killing vectors. In particular, we are interested in finding the horizon properties associated with the outer horizon, the horizon causally connected to the $\mathrm{AdS}_{3}$ boundary. The Killing vector generating this outer (event) horizon should remain time-like in the $r^{2}>r_{\mathcal{H}}^{2}$ region. This outer horizon can be generated by $\zeta_{\mathcal{H}_{+}}\left(\zeta_{\mathcal{H}_{-}}\right)$in the regions where $\psi_{1} \psi_{2} \phi_{1} \phi_{2}$ is negative (positive). If the outer horizon is generated by $\zeta_{\mathcal{H}_{+}}\left(\zeta_{\mathcal{H}_{-}}\right)$the horizon radius is $r_{\alpha+}^{2}\left(r_{\alpha-}^{2}\right)$, where $r^{2}=r_{1+}^{2}$ and $r^{2}=r_{2+}^{2}\left(r^{2}=r_{1-}^{2}\right.$ and $\left.r^{2}=r_{2-}^{2}\right)$ denote the 
Table 3 Left and right top tables, respectively, show, the signs of $r_{1+}^{2}-r_{1-}^{2}$ and $r_{1+}^{2}-r_{2-}^{2}$. Left and right bottom tables, respectively, show the signs of $r_{2+}^{2}-r_{1-}^{2}$ and $r_{2+}^{2}-r_{2-}^{2}$. To deduce the above tables we have used (2.16)

\begin{tabular}{|c|c|c|c|c|c|c|c|c|c|c|c|}
\hline$x^{+} x^{-}$ & $I_{1, j}^{-}$ & $I_{2, j}^{-}$ & $I_{3, j}^{-}$ & $I_{4, j}^{-}$ & $I_{1, j+1}^{-}$ & $x^{+}$ & $I_{1, j}^{-}$ & $I_{2, j}^{-}$ & $I_{3, j}^{-}$ & $I_{4, j}^{-}$ & $I_{1, j+1}^{-}$ \\
\hline$I_{1, i}^{+}$ & + & - & - & - & + & $I_{1, i}^{+}$ & + & + & - & - & + \\
\hline$I_{2, i}^{+}$ & + & - & - & - & + & $I_{2, i}^{+}$ & - & - & + & + & - \\
\hline$I_{3, i}^{+}$ & - & + & + & + & - & $I_{3, i}^{+}$ & - & - & + & + & - \\
\hline$I_{4, i}^{+}$ & - & + & + & + & - & $I_{4, i}^{+}$ & - & - & + & + & - \\
\hline$I_{1, i+1}^{+}$ & + & - & - & - & + & $I_{1, i+1}^{+}$ & + & + & - & - & + \\
\hline$x^{+} x^{-}$ & $I_{1, j}^{-}$ & $I_{2, j}^{-}$ & $I_{3, j}^{-}$ & $I_{4, j}^{-}$ & $I_{1, j+1}^{-}$ & & $I_{1, j}^{-}$ & $I_{2, j}^{-}$ & $I_{3, j}^{-}$ & $I_{4, j}^{-}$ & $I_{1, j+1}^{-}$ \\
\hline$I_{1, i}^{+}$ & + & - & - & + & + & $I_{1, i}^{+}$ & + & - & - & - & + \\
\hline$I_{2, i}^{+}$ & - & + & + & - & - & $I_{2, i}^{+}$ & - & + & + & + & - \\
\hline$I_{3, i}^{+}$ & - & + & + & - & - & $I_{3, i}^{+}$ & - & + & + & + & - \\
\hline$I_{4, i}^{+}$ & - & + & + & - & - & $I_{4, i}^{+}$ & + & - & - & - & + \\
\hline$I_{1, i+1}^{+}$ & + & - & - & + & + & $I_{1, i+1}^{+}$ & + & - & - & - & + \\
\hline
\end{tabular}

two branches of the bifurcate horizon. We will return to this point later.

To fix the normalization of the horizon generating Killing vectors, we focus on the regions where $\psi_{1} \psi_{2}$ and $\phi_{1} \phi_{2}$ are both positive, and hence the event horizon is generated by $\zeta_{\mathcal{H}_{+}}$. A similar analysis may also be repeated for the other regions, including cases with negative $\Pi$, where the outer (event) horizon is generated by $\zeta_{\mathcal{H}_{-}}$. Let us study the large $r$, asymptotic behavior of the Killing vector $\zeta_{\mathcal{H}_{+}}$. One may readily see from (2.7) that at large $r$

$\zeta_{+} \sim K_{+} \partial_{+}, \quad \zeta_{-} \sim K_{-} \partial_{-}$.

The "appropriate" normalization is hence the one in which $\zeta_{ \pm}$are along the coordinates. Noting (2.10) and (2.12) we learn that

$\zeta_{+} \sim \frac{1}{2 \mathcal{T}_{+}} \partial_{X^{+}}, \quad \zeta_{-} \sim \frac{1}{2 \mathcal{T}_{-}} \partial_{X^{-}}$

where

$X^{+}=\frac{1}{2 \mathcal{T}_{+}} \ln \frac{\psi_{1}}{\psi_{2}}, \quad X^{-}=\frac{1}{2 \mathcal{T}_{-}} \ln \frac{\phi_{1}}{\phi_{2}}$.

In the above the normalization factors $\frac{1}{2 \mathcal{T}_{ \pm}}$are chosen recalling (2.11), such that $X^{ \pm}$are $2 \pi$ periodic; explicitly, $X^{ \pm}\left(x^{ \pm}+2 \pi\right)=X^{ \pm}\left(x^{ \pm}\right)+2 \pi$. The appropriate asymptotic time and angular variable, $\tau, \varphi$ are hence

$\tau=\ell\left(X^{+}+X^{-}\right) / 2, \quad \varphi=\left(X^{+}-X^{-}\right) / 2$.

With the above normalization and recalling (2.21), we learn that the physical surface gravity $\kappa$ is
$\frac{2}{\kappa}=\frac{1}{\mathcal{T}_{+}}+\frac{1}{\mathcal{T}_{-}}$

$\mathcal{H}_{1 \pm}, \mathcal{H}_{2 \pm}$ intersecting the boundary, number of disconnected pieces at the boundary Let us focus on the regions where $\psi_{1} \psi_{2}$ and $\phi_{1} \phi_{2}$ are both positive, and hence the event horizon is generated by $\zeta_{\mathcal{H}_{+}}$. A similar analysis may also be repeated for the other regions. The "horizon radii" $r_{\alpha+}^{2}$ can range from minus infinity to plus infinity. The places $r_{1+}^{2}$ or $r_{2+}^{2}$ that become infinite is where the horizon $\mathcal{H}_{1+}$ or $\mathcal{H}_{2+}$ intersect the $\mathrm{AdS}_{3}$ boundary. To be explicit, in our $5 \times 5$ tables, the segments of the boundary are in the $(1,1),(2,2)$ and in $(4,4),(5,5)$ parts of the table. From the tables we also see that there are $(3,3)$ and parts of $(2,2),(4,4)$ regions where $\zeta_{\mathcal{H}_{+}}$can have zeros at $r^{2}<r_{0}^{2}$ [cf. (2.3)].

In fact, one can readily see that the extrema of the functions $r_{\alpha \pm}^{2}$ occur at $^{7}$

$\partial_{+} r_{\alpha \pm}^{2}=0, \partial_{-} r_{\alpha \pm}^{2}=\left.0 \Longrightarrow r_{\alpha \pm}^{2}\right|_{\text {extremum }}=r_{0}^{2}$.

The extrema are minima in regions where $r_{\alpha \sigma}^{2}$ can become very large (and become infinite at the boundaries of the region) and maximum in the regions where at its boundaries $r_{\alpha \sigma}^{2}$ can become zero. That is, in region $(3,3)$ we have a maximum; in regions $(1,1),(5,5)$ we have a minimum and in regions $(2,2)$ and $(4,4)$ we have a maximum and a minimum. One can show (e.g. see Appendix A for the case of BTZ black holes) that the $r^{2}>r_{0}^{2}$ and $0<r^{2}<r_{0}^{2}$ regions are geometrically the same, corresponding to the regions I and I' on the Penrose diagram in Fig. 2. The Bañados coor-

7 Note that at $\left(\frac{\psi^{\prime}}{\psi}\right)^{\prime}=0,\left(\frac{\psi^{\prime}}{\psi}\right)^{2}=L_{+}$, and similarly for $\phi$ and hence
the extremum of $\frac{\psi^{\prime}}{\psi} \frac{\phi^{\prime}}{\phi}$ occurs at $\sqrt{L_{+} L_{-}}$. 
dinates cover these parts twice. So, we would not count the event horizons appearing in the $0<r^{2}<r_{0}^{2}$ region as independent.

We next note that in the region $\Pi<0$, the outer horizon may be generated by $\zeta_{\mathcal{H}_{-}}$. In these regions $r_{1-}^{2}$ or $r_{2-}^{2}$ may become infinite. In terms of our $5 \times 5$ tables, this happens at the boundaries of $(4,1)$ and $(5,2)$ regions (where $r_{1-}^{2}$ becomes infinite) and at the boundaries of $(1,4)$ and $(2,5)$ (where $r_{2-}^{2}$ becomes infinite). The regions $(2,3),(3,4)$ and $(3,2),(4,3)$ are again a repetition of these parts and we do not count them separately.

Summarizing, the above analysis shows that we have $\left(n_{+}+1\right)\left(n_{-}+1\right)$ causally disconnected regions in the range $x^{ \pm} \in[0,2 \pi]$ at the boundary. These regions are separated by the roots of $\psi_{1}^{\prime}, \psi_{2}^{\prime}, \phi_{1}^{\prime}$ and $\phi_{2}^{\prime}$. Note that this is exactly the place where $\left|\zeta_{\mathcal{H}_{ \pm}}\right|^{2}$ around the small $r$ region changes sign. It is also instructive to recall the case of BTZ black holes and in particular Fig. 2.

Regions enclosed by the boundary and the event horizon Let us consider the case where the outer horizon is generated by $\zeta_{\mathcal{H}_{+}}$, i.e. where $\Pi>0$. The two surfaces $r^{2}=r_{1+}^{2}$ and $r^{2}=r_{2+}^{2}$ define the two branches of the bifurcate (event) horizon. ${ }^{8}$ As discussed there are also patches defined by the intersection of $\mathcal{H}_{1-}$ or $\mathcal{H}_{2-}$ and the boundary (in the $\Pi<0$ regions). There are $\left(n_{+}+1\right)\left(n_{-}+1\right)$ causally disconnected regions bordered by the two branches of the event horizon and the boundary.

Bifurcation curve $\Sigma$ As in the previous discussion, let us focus on the regions where $\psi_{1} \psi_{2}$ and $\phi_{1} \phi_{2}$ are both positive, and hence the event horizon is generated by $\zeta_{\mathcal{H}_{+}}$. A similar analysis may also be repeated for the other regions. As mentioned the $\mathcal{H}_{1+}$ and $\mathcal{H}_{2+}$ are two-dimensional null surfaces generated by the Killing vector field $\zeta_{\mathcal{H}_{+}}$. In the terminology of the usual Penrose diagram or Kruskal coordinates, we introduced $u, v$ null coordinates, that is, $\mathcal{H}_{1+}$ and $\mathcal{H}_{2+}$ are along constant $u$ and constant $v$ surfaces. They can intersect on a space-like one-dimensional curve $\Sigma$ (which is at $u=v=0$ ). In the usual terminology $\Sigma_{+}$is the bifurcation curve. Similarly to $\mathcal{H}_{1+}$ and $\mathcal{H}_{2+}, \Sigma_{+}$is not necessarily simply connected and may have some disconnected pieces. Below we discuss some properties of the bifurcation curve $\Sigma_{+}$:

1. The Killing vector field which generates the horizon vanishes at $\Sigma_{+}$. In the region we are discussing the horizon is generated by $\zeta_{\mathcal{H}_{+}}$and

\footnotetext{
${ }^{8}$ In the BTZ case $r_{1+}^{2}=r_{2+}^{2}=r_{+}^{2}$, and as usual on the Penrose diagram (see Fig. 2) the event horizon is specified by $r=r_{+}$. Note, however, the difference between the BTZ and the Bañados coordinate systems ( $c f$. Appendix A.1).
}

$\left.\zeta_{\mathcal{H}_{+}}\right|_{\Sigma_{+}}=0$

This may be checked by a straightforward computation at $\Sigma_{+}=\mathcal{H}_{1+} \cap \mathcal{H}_{2+}$. To this end, it is useful to note that at $\Sigma_{+}$:

$\Sigma_{+}: \quad \psi_{1}^{\prime} \psi_{2}=-\phi_{2}^{\prime} \phi_{1}, \quad \psi_{2}^{\prime} \psi_{1}=-\phi_{1}^{\prime} \phi_{2}$

In a similar way, one may check that the above is also true when the horizon is generated by $\zeta_{\mathcal{H}_{-}}$with the bifurcation curve $\Sigma_{-}, \Sigma_{-}=\mathcal{H}_{1-} \cap \mathcal{H}_{2-}$. For the $\Sigma_{-}$, however, we have

$\Sigma_{-}: \quad \psi_{1}^{\prime} \psi_{2}=\phi_{1}^{\prime} \phi_{2}, \quad \psi_{2}^{\prime} \psi_{1}=\phi_{2}^{\prime} \phi_{1}$

2. $\Sigma_{ \pm}$is along the Killing vector $\zeta_{\mathcal{H}_{\mp}}$ One can readily show that the tangent to the curve at the intersection of $r^{2} \equiv$ $r_{\mathcal{H}_{1 \pm}}^{2}$ and $r^{2} \equiv r_{\mathcal{H}_{2 \pm}}^{2}$ surfaces is along $\zeta_{\mathcal{H}_{\mp}}$. In other words, $\Sigma_{ \pm}$is generated by the flow of $\zeta_{\mathcal{H}_{\mp}}$ at $\mathcal{H}_{1 \pm}$ or $\mathcal{H}_{2 \pm}$. Moreover, recalling (2.17), we see that $\zeta_{\mathcal{H}_{\mp}}$ are space-like at $\Sigma_{ \pm}$.

3. One may calculate the Bañados metric (2.2) at $\Sigma_{+}$to obtain

$$
\begin{aligned}
\left.\mathrm{d} s^{2}\right|_{\Sigma_{+}} & =\ell^{2} \mathrm{~d} \Phi_{+}^{2}, \\
\Phi_{+} & =\frac{1}{2} \ln \frac{\psi_{1}^{\prime} \psi_{1}}{\phi_{1}^{\prime} \phi_{1}}=-\frac{1}{2} \ln \frac{\psi_{2}^{\prime} \psi_{2}}{\phi_{2}^{\prime} \phi_{2}} .
\end{aligned}
$$

To show the second equality above we have used (2.29). The above, together with (2.17), implies that $\zeta_{\mathcal{H}_{-}} \mid \Sigma_{+}=$ $\partial_{\Phi_{+}}$.

If the outer horizon is generated by $\zeta_{\mathcal{H}_{-}}$, with the bifurcation curve $\Sigma_{-}$, the metric at $\Sigma_{-}$is given by

$$
\left.\mathrm{d} s^{2}\right|_{\Sigma_{-}}=\ell^{2} \mathrm{~d} \Phi_{-}^{2}, \Phi_{-}=\frac{1}{2} \ln \frac{\psi_{1}^{\prime} \psi_{1}}{\phi_{2}^{\prime} \phi_{2}}=-\frac{1}{2} \ln \frac{\psi_{2}^{\prime} \psi_{2}}{\phi_{1}^{\prime} \phi_{1}} .
$$

The coordinate $\Phi_{-}$is along the Killing vector $\zeta_{\mathcal{H}_{+}}$.

4. Using (2.11) we learn that

$$
\Phi_{ \pm}=\mathcal{T}_{+} x^{+} \mp \mathcal{T}_{-} x^{-}+\mathcal{P}_{ \pm}\left(x^{+}\right)+\mathcal{Q}_{ \pm}\left(x^{-}\right),
$$

where $\mathcal{P}_{ \pm}, \mathcal{Q}_{ \pm}$are periodic functions, $\mathcal{P}_{ \pm}\left(x^{+}\right)=$ $\mathcal{P}_{ \pm}\left(x^{+}+2 \pi\right), \mathcal{Q}_{ \pm}\left(x^{-}\right)=\mathcal{Q}_{ \pm}\left(x^{-}+2 \pi\right)$.

5. Horizon angular velocity From (2.32) and (2.24) we learn that

$$
\Phi_{ \pm}=R_{\mathcal{H}}^{ \pm}\left(\varphi-\Omega_{\mathcal{H}}^{ \pm} \frac{\tau}{\ell}\right)+\text { "periodic part", }
$$


where

$$
R_{\mathcal{H}}^{ \pm}=\mathcal{T}_{+} \pm \mathcal{T}_{-}, \quad \Omega_{\mathcal{H}}^{+}=\frac{1}{\Omega_{\mathcal{H}}^{-}}=\frac{\mathcal{T}_{+}-\mathcal{T}_{-}}{\mathcal{T}_{+}+\mathcal{T}_{-}}
$$

One may readily show that we have the same horizon angular velocity if we considered the event horizon generated by $\zeta_{\mathcal{H}}$ and the bifurcation curve $\Sigma_{-} .{ }^{9}$

6. Length of $\Sigma_{+}$, "area" of horizon As can be explicitly seen from (2.30) and (2.33), $\Sigma$ is a curve of finite length,

$\mathcal{A}_{\mathcal{H}}=2 \pi \ell\left(\mathcal{T}_{+}+\mathcal{T}_{-}\right)$

One may readily see that $\Sigma_{-}$has the same "length" as above.

7. Bifurcation curve $\Sigma$ has $\left(n_{+}+1\right)\left(n_{-}+1\right)$ disconnected pieces The bifurcation curves $\Sigma_{+}$, as parametrized by $\Phi_{+}$ coordinate (2.32), consist of some disconnected pieces. To distinguish the disconnected pieces, we should check where the equations defining $\Sigma_{+}$(2.29) have solutions and also where $\Phi$ is real-valued. Given the information in our tables, we learn that $\Sigma_{+}$has the same number of disconnected pieces as the $\mathcal{H}_{\alpha+}$. Considering the outer (event) horizons generated by $\zeta_{\mathcal{H}_{+}}$, the bifurcation curve of the event horizon has $\left(n_{+}+1\right)\left(n_{-}+1\right)$ disconnected pieces.

Inner horizons Associated with any event horizon there is, generically, an inner horizon. The region "inside" the inner horizon region is bounded between the inner horizon (which is where we have a null Killing vector field) and the region we develop a CTC. Inside this region the horizon generating Killing vector should remain time-like. When the event horizon is generated by $\zeta_{\mathcal{H}_{ \pm}}$the inner (Cauchy) horizon is generated by $\zeta_{\mathcal{H}_{\mp}}$. We note that, as is seen in Table 3 , in the regions the event horizon is given by $r_{2+}^{2}\left(r_{1+}^{2}\right), r_{2+}^{2}-r_{2-}^{2}$, and $r_{2+}^{2}-r_{1-}^{2}\left(r_{1+}^{2}-r_{1-}^{2}\right.$ and $\left.r_{1+}^{2}-r_{2-}^{2}\right)$ are both positive, confirming the picture that outer horizons occur at a larger $r$-coordinate value than the inner horizon. This statement is also true when the event (outer) horizons are generated by $\zeta_{\mathcal{H}_{-}}$.

As commented in footnote 9 , regardless of whether the Killing horizon generating the inner horizon is $\zeta_{\mathcal{H}_{\mp}}$, one may show that the coordinate along the inner horizon is $\Phi_{-}$[cf. (2.30), (2.31)].

Summary of subsection We argued that the two Killing vectors $\zeta_{\mathcal{H}_{ \pm}}$which are everywhere orthogonal can become null

\footnotetext{
${ }^{9}$ Note that if the event horizon is generated by $\zeta_{\mathcal{H}_{-}}$, one should revisit the definition of $X^{ \pm}$(2.24), by taking either of $X^{-}$or $X^{+}$to minus themselves. In this way the coordinate covering the event horizon will always keep the form $\Phi_{+}$. As we will discuss, $\Phi_{-}$will be the coordinate on the inner horizon.
}

on some different patches of spacetime. These null surfaces can intersect the boundary in different places. As discussed boundary and horizon, in general, have $\left(n_{+}+1\right)\left(n_{-}+1\right)$ disconnected pieces. When the outer horizon is generated by $\zeta_{\mathcal{H}_{ \pm}}$the corresponding inner horizon is generated by $\zeta_{\mathcal{H}_{\mp}}$. Regardless of which $\zeta$ produces the horizon, the coordinate spanning the bifurcation curve of the event horizon is $\Phi_{+}$ and that of the inner horizon is $\Phi_{-}$. The length of the event horizon is always $2 \pi \ell R_{\mathcal{H}}^{+}$and that of the inner horizon is $2 \pi \ell R_{\mathcal{H}}^{-}$. We have summarized the above information in the Penrose diagram Fig. 1.

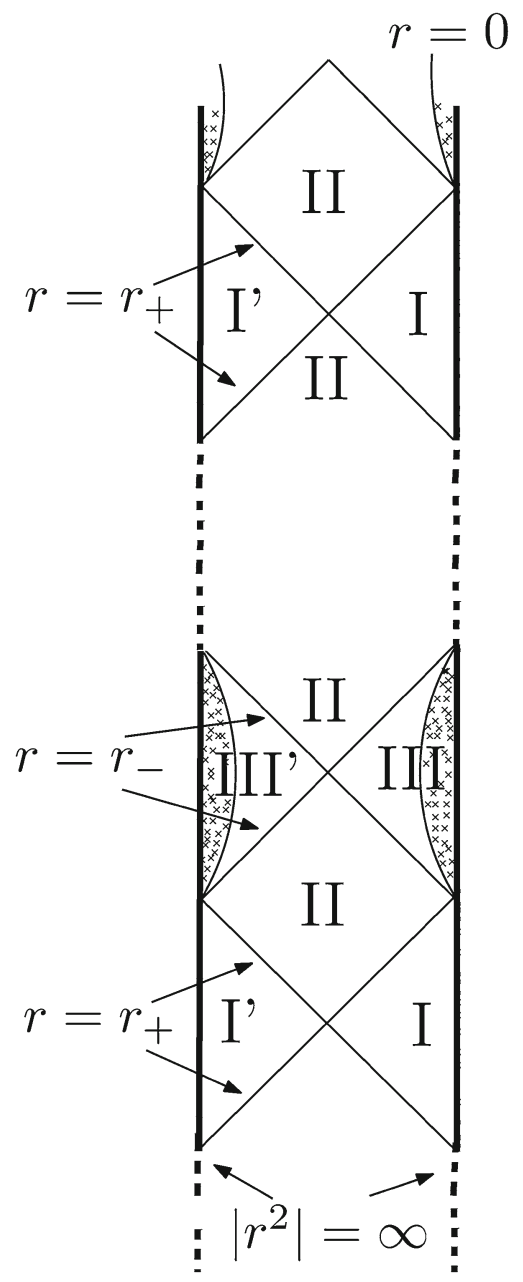

Fig. 1 Penrose diagram for generic Bañados geometry. To draw this causal diagram we have used the analysis of the simpler case of BTZ (cf. Appendix A.1) and the analysis made in this section. In the Penrose diagram, as usual, we have suppressed a space-like compact direction (here the one along $\Phi_{+}$or $\Phi_{-}$coordinates). Our discussions in this section reveal that Penrose diagrams for generic $n_{ \pm}$are essentially the same as those of usual BTZ geometries discussed in $[7,8]$. However, we cut the regions which have CTCs and we should make appropriate identifications, and hence we are left with a geometry whose Penrose diagram is an $\left(n_{+}+1\right)\left(n_{-}+1\right)$ multiple repetition of that of a single BTZ geometry (Fig. 2) 


\subsection{Geodesic motion on generic Bañados geometries}

To gain a better intuition about the Bañados geometries we present a brief analysis of geodesic motion on these backgrounds. A similar study has been performed for constant $L_{ \pm}$case, cases which include BTZ black holes, in the literature. For clarity and completeness we have presented those analyses in Appendix A.2. Here, we give a discussion for generic $L_{ \pm}$functions.

As discussed, any Bañados geometry generically has two global Killing vectors $\zeta_{ \pm}$and four local ones, and altogether we have six Killing vectors which form a $\operatorname{sl}(2, \mathbb{R}) \times \operatorname{sl}(2, \mathbb{R})$ algebra. We may use these facts to construct the geodesics. Let us consider the geodesic velocity vector $v^{\mu}=d x^{\mu} / d s$, where $s$ is the affine parameter on the geodesic. The velocity along the Killing vectors is a constant of motion. Therefore, $P_{ \pm}$

$P_{ \pm}=v \cdot \zeta_{\mathcal{H}_{ \pm}}$

are constants of motion. Having two constants of motion, we can completely solve for the velocity vector using the fact that

$v^{2}=\sigma, \sigma=-1,0,+1$

respectively, for time-like, null, and space-like geodesics.

To write out the above equation explicitly, let us define an orthogonal basis, $\zeta_{\mathcal{H}_{+}}$and $\eta$, with

$\ell^{-1} \eta=\eta^{r} \partial_{r}+\eta^{+} \partial_{+}+\eta^{-} \partial_{-}$,

where $\eta \cdot \zeta_{\mathcal{H}_{ \pm}}=0$ and

$$
\begin{aligned}
\eta^{r}= & -\frac{K_{+} K_{-}}{\ell^{2} r}\left(r^{4}-r_{\mathcal{H}_{1}}^{2} r_{\mathcal{H}_{2}}^{2}\right), \\
\eta^{ \pm}= & K_{ \pm} K_{\mp}^{\prime} \\
& -\frac{\ell^{2} r^{2} K_{ \pm}^{\prime}\left(K_{\mp}^{\prime \prime}-4 L_{\mp} K_{\mp}\right)+\ell^{4} K_{\mp}^{\prime}\left(K_{ \pm}^{\prime \prime}-4 L_{ \pm} K_{ \pm}\right)}{2\left(r^{4}-l^{4} L_{+} L_{-}\right)},
\end{aligned}
$$

and one may show that

$|\eta|^{2}=-\left|\zeta_{\mathcal{H}_{+}}\right|^{2}\left|\zeta_{\mathcal{H}_{-}}\right|^{2}$

We can then expand the velocity vector $v$ as

$v=\frac{P_{+}}{\left|\zeta_{\mathcal{H}_{+}}\right|^{2}} \zeta_{\mathcal{H}_{+}}+\frac{P_{-}}{\left|\zeta_{\mathcal{H}_{-}}\right|^{2}} \zeta_{\mathcal{H}_{-}}+\frac{Z}{|\eta|^{2}} \eta$,

where $Z=v \cdot \eta$ and

$$
\begin{aligned}
Z^{2} & =|\eta|^{2}\left(\sigma-\frac{P_{+}^{2}}{\left|\zeta_{\mathcal{H}_{+}}\right|^{2}}-\frac{P_{-}^{2}}{\left|\zeta_{\mathcal{H}_{-}}\right|^{2}}\right) \\
& =|\eta|^{2} \sigma+P_{+}^{2}\left|\zeta_{\mathcal{H}_{-}}\right|^{2}+P_{-}^{2}\left|\zeta_{\mathcal{H}_{+}}\right|^{2} .
\end{aligned}
$$

What will be important in our further discussions in Sects. 4 and 5 is that $Z^{2}, P_{ \pm}$are diffeomorphism invariant quantities. Therefore, geodesic observers can only probe a part of the information encoded in the functions $L_{ \pm}$, which specify the geometric properties of the background.

\subsection{Summary of Bañados geometries and outlook of the section}

We argued that in general, irrespective of the details of the functions $L_{ \pm}$, Bañados metrics (2.2) have $U(1)_{+} \times U(1)_{-}$ global isometries. The corresponding Killing vectors may be constructed through the solutions to the Hill equations (2.9), the $\psi$ and $\phi$ functions. In general, for nonconstant $L_{ \pm}$ cases, the norm of the Killing vector fields $\zeta_{\mathcal{H}_{+}}$can vanish at four surfaces given in (2.15). As discussed two of these four surfaces provide the outer (event) bifurcate horizon, while the other two lead to the bifurcate inner Killing horizon. The Bañados coordinate system, once the range $r^{2}<0$ is also included, covers the regions outside the outer horizon (all the way to the boundary) and the inside the inner horizon (all the way to the CTC region). It does not necessarily cover the (whole) region between the two horizons. Moreover, as discussed, in the allowed region for $r^{2}$, it provides a double cover of the part of spacetime in covers. ${ }^{10}$

As discussed in some detail, in the $x^{ \pm} \in[0,2 \pi]$ range the horizons intersect the boundary in $\left(n_{+}+1\right)\left(n_{-}+1\right)$ distinct regions, where $n_{+}, n_{-}$are, respectively, the number of zeros of the $\psi$ and $\phi$ functions. Moreover, we showed that the inner and outer bifurcation curves $\Sigma_{ \pm}$also have the same number of disconnected pieces. The surface gravity $\kappa$, angular velocity $\Omega_{\mathcal{H}}$, and length $A_{\mathcal{H}}$ of the outer horizon are given in terms of the Floquet indices of the $\psi$ and $\phi$ solutions, $\mathcal{T}_{ \pm}$, as

$$
\begin{gathered}
\frac{2}{\kappa}=\frac{1}{\mathcal{T}_{+}}+\frac{1}{\mathcal{T}_{-}}, \quad \Omega_{\mathcal{H}}=\frac{\mathcal{T}_{+}-\mathcal{T}_{-}}{\mathcal{T}_{+}+\mathcal{T}_{-}}, \\
A_{\mathcal{H}}=2 \pi \ell\left(\mathcal{T}_{+}+\mathcal{T}_{-}\right) .
\end{gathered}
$$

There is a closely related construction for multi-BTZ geometries due to Brill [27-29]. This construction is based on the fact that a constant time slice for $\mathrm{AdS}_{3}$ has the line element $d s^{2}=\left(d z^{2}+d x^{2}\right) / z^{2}$, which is nothing but a 2 d hyperboloid $H^{2}$. The latter may also be viewed as the Poincaré disk. It is well known that one may construct all $2 \mathrm{~d}$ surfaces of genus $g \geq 2$ from orbifolds of $H^{2}$, by making identifications on it with a discrete subgroup of its $\operatorname{sl}(2, \mathbb{R})$ isometry. It is then argued that if we add back the time direction, the $3 \mathrm{~d}$ geometry we obtain in this way is a black hole geometry with a multisector horizon and boundary. In a different terminology, one

\footnotetext{
${ }^{10}$ It is instructive to see the analysis of Appendix A.1 for the simpler case of constant $L_{ \pm}$.
} 
may cut through the maximally extended multi-BTZ geometry at the constant time slice passing through the horizon. In this way, one observes that the multi-BTZ geometry is indeed a wormhole with a number of handles $g[30,31]$.

In the "Brill diagrams" [27-29], the construction is based upon a time-direction suppressed $3 \mathrm{~d}$ geometry. In our tables, we discuss the $x^{ \pm}$, while suppressing the radial direction $r$ and in a Penrose diagram (see Fig. 1) we are suppressing the "compact $\Phi$ " direction. In a sense these three are complementary to each other. Making a thorough comparison and working out the details of connection between our multi-sector geometries and those of Brill is postponed to upcoming work.

\section{Bañados geometries and the associated conserved charges}

As was discussed in [12], to a given Bañados geometry one may associate two kind of charges, the Virasoro charges and the charges associated to Killing vectors $\zeta_{ \pm}$. It was shown in [12] that the usual Lee-Wald [32] or Barnich-Brandt [33] symplectic structure vanishes on-shell for Bañados geometries, and hence within the covariant phase space method (see e.g. [16] for a review) one can define symplectic symmetries. That is, the surface charges could be defined by integration on a space-like one-dimensional curve everywhere in the geometry and not necessarily at the boundary.

It was argued in [16] that symplectic symmetries as defined above, may be in either of the two groups: The generator of the symmetry could be an exact symmetry, in our setting a Killing vector, or it can be a nontrivial diffeomorphism which is not an exact symmetry. The former may hence be called a "symplectic exact symmetry" (SES) and the latter a "symplectic non-exact symmetry" (SNS). In our example the SNSs are generated by diffeomorphisms along the $\chi$ vector field (2.5) and the SESs are generated by the Killing vectors $\zeta_{ \pm}(2.7)$ [12].

In this section we give the expression for the charges associated with SNS and SES. The first part of this section is essentially a review of [12]. However, the second part is a completion of [12] along the analysis of [16].

\subsection{Charges associated with symplectic non-exact symmetries ( $\mathrm{SNSs}$ )}

In the covariant phase space method, we first define charge variations associated with field variations/perturbations generated by a vector field, e.g. $\chi$, and then we integrate these charge variations over a given path in the phase space to define the charge itself. The integrability condition, which is checked to hold in our case [12], then guarantees that the charges do not depend on the integration path in the phase space.

For the Bañados geometry with $L_{ \pm}$functions, if we denote the charges associated with $\chi\left[\epsilon_{+}=e^{i n x^{+}}, \epsilon_{-}=0\right]$ and $\chi\left[\epsilon_{+}=0, \epsilon_{-}=e^{i n x^{-}}\right]$[cf. (2.5)], respectively, by $L_{n}, \bar{L}_{n}$, their explicit form is [12]

$$
\begin{aligned}
L_{n}[g] & =\frac{\ell}{8 \pi G} \oint \mathrm{d} x^{+} L_{+}\left(x^{+}\right) e^{i n x^{+}}, \\
\bar{L}_{n}[g] & =\frac{\ell}{8 \pi G} \oint \mathrm{d} x^{-} L_{-}\left(x^{-}\right) e^{i n x^{-}} .
\end{aligned}
$$

These charges form two Virasoro algebras at the BrownHenneaux central charge:

$$
\begin{aligned}
& {\left[L_{n}, L_{m}\right]=(n-m) L_{n+m}+\frac{c}{12} \delta_{m+n} n^{3},} \\
& {\left[L_{n}, \bar{L}_{m}\right]=0, \quad\left[\bar{L}_{n}, \bar{L}_{m}\right]=(n-m) \bar{L}_{n+m}+\frac{c}{12} \delta_{m+n} n^{3},}
\end{aligned}
$$

with

$c=\frac{3 \ell}{2 G}$,

where $G$ is the $3 \mathrm{~d}$ Newton constant. Note that unlike the usual Brown-Henneaux case [9], (1) our charges are defined everywhere and not just close to the boundary; (2) the charges are defined around an arbitrary Bañados solution and not just $\mathrm{AdS}_{3}$ (in Poincaré patch). This latter item brings about two interesting features: first, the charges are associated with each Bañados geometry and, second, the charges become field dependent, their expression explicitly depends on the background functions $L_{ \pm}$. Therefore, to obtain the algebra of charges one needs to consider an "adjusted bracket", which includes the change made the background functions when computing charge variations; see [12] for detailed discussions and analysis.

\subsection{Charges associated with symplectic exact symmetries} (SESs)

In [12] it has been argued that one can associate the following conserved charges to $\mathrm{U}(1)_{ \pm}$Killing vectors of the geometry (2.2). Using the analysis of [16], we obtain ${ }^{11}$

$\delta J_{ \pm}=\frac{\ell}{8 \pi G} \int_{0}^{2 \pi} \mathcal{K}_{ \pm}^{0} \delta L_{ \pm} \mathrm{d} x^{ \pm}$

where, using the Floquet form of the solutions (2.11) and (2.12),

$\mathcal{K}_{+}^{0}=2 \mathcal{T}_{+} \cdot \psi_{1}\left(x^{+} ; \mathcal{T}_{+}\right) \psi_{2}\left(x^{+} ; \mathcal{T}_{+}\right)$,

$\mathcal{K}_{-}^{0}=2 \mathcal{T}_{-} \cdot \phi_{1}\left(x^{-} ; \mathcal{T}_{-}\right) \phi_{2}\left(x^{-} ; \mathcal{T}_{-}\right)$

$\overline{11}$ To simplify the notation and avoid cluttering here we use $\delta X$ for denoting what was called parametric variations in [16]. In the latter paper, this was denoted by $\hat{\delta} X$. 
and

$\delta L_{ \pm}=\frac{\partial L_{ \pm}}{\partial \mathcal{T}_{ \pm}} \delta \mathcal{T}_{ \pm}$.

The numeric coefficients $2 \mathcal{T}_{ \pm}$in (3.5) are twice the Floquet index (2.11) and have been added recalling the discussions surrounding (2.24) in Sect. 2.3.2.

Using (2.9) and (2.10) we learn that

$$
\begin{aligned}
\mathcal{K}_{+}^{0} \delta L_{+} & =2 \mathcal{T}_{+}\left(\psi_{2} \delta \psi_{1}^{\prime \prime}-\psi_{2}^{\prime \prime} \delta \psi_{1}\right) \\
& =2 \mathcal{T}_{+}\left(\psi_{2} \delta \psi_{1}^{\prime}-\psi_{2}^{\prime} \delta \psi_{1}\right)^{\prime},
\end{aligned}
$$

and similarly for the right-movers (the case labeled by a minus index). In the above a prime denotes derivative w.r.t. the argument $x^{ \pm}$and $\delta$ denotes variations w.r.t. the Floquet index $\mathcal{T}_{ \pm}$. Therefore,

$\delta J_{+}=\frac{\ell \mathcal{T}_{+}}{4 \pi G}\left[\psi_{2} \delta \psi_{1}^{\prime}-\psi_{2}^{\prime} \delta \psi_{1}\right]_{0}^{2 \pi}$,

$\delta J_{-}=\frac{\ell \mathcal{T}_{-}}{4 \pi G}\left[\phi_{2} \delta \phi_{1}^{\prime}-\phi_{2}^{\prime} \delta \phi_{1}\right]_{0}^{2 \pi}$.

One may now use the Floquet form (2.11) and the normalization condition (2.10) to further simplify the above:

$\left[\psi_{2} \delta \psi_{1}^{\prime}-\psi_{2}^{\prime} \delta \psi_{1}\right]_{0}^{2 \pi}=\left[x^{+} \delta \mathcal{T}_{+}\right]_{0}^{2 \pi}=2 \pi \delta \mathcal{T}_{+}$,

$\left[\phi_{2} \delta \phi_{1}^{\prime}-\phi_{2}^{\prime} \delta \phi_{1}\right]_{0}^{2 \pi}=\left[x^{-} \delta \mathcal{T}_{-}\right]_{0}^{2 \pi}=2 \pi \delta \mathcal{T}_{-}$.

To get the charges $J_{ \pm}$we need to integrate over $\delta J_{ \pm}$on path in the $\mathcal{T}_{ \pm}$space, i.e.

$J_{ \pm}=\frac{\ell}{2 G} \int_{\mathcal{T}_{ \pm 0}}^{\mathcal{T}_{ \pm}} \mathrm{d} \tilde{\mathcal{T}}_{ \pm} \tilde{\mathcal{T}}_{ \pm}=\frac{\ell}{4 G}\left(\mathcal{T}_{ \pm}^{2}-\mathcal{T}_{ \pm 0}^{2}\right)=\frac{c}{6}\left(\mathcal{T}_{ \pm}^{2}-\mathcal{T}_{ \pm 0}^{2}\right)$,

where $\mathcal{T}_{ \pm 0}$ is a reference point which has zero $J_{ \pm}$. As we will discuss in the next section two standard choices are $\mathcal{T}_{ \pm 0}^{2}=$ $-1 / 4$ (when $J_{ \pm}$are measured w.r.t. global $\mathrm{AdS}_{3}$ ) or $\mathcal{T}_{ \pm 0}^{2}=0$ when the reference point is chosen as $\mathrm{AdS}_{3}$ in a Poincare patch (massless BTZ).

The very important point discussed in [12] is that the $J_{ \pm}$ charges above commute with the Virasoro generators $L_{n}, \bar{L}_{n}$,

$\left[J_{ \pm}, L_{n}\right]=\left[J_{ \pm}, \bar{L}_{n}\right]=0, \quad \forall n \in \mathbb{Z}$.

$B T Z$ case For the BTZ black hole solution, where $L_{ \pm}=\mathcal{T}_{ \pm}^{2}$ and $\mathcal{T}_{ \pm 0}^{2}=0$, we get

$J_{ \pm}=\frac{\ell}{4 G} \mathcal{T}_{ \pm}^{2}=\ell M_{\mathrm{BTZ}} \pm J_{\mathrm{BTZ}}$

Also, in this case the normalization factor $2 \mathcal{T}_{ \pm}$is the temperature in the left and right moving sectors.
3.3 Entropy, the first law and Smarr relation for Bañados geometries

As in the standard Wald formulation $[34,35]$ the charge associated with the outer (event) Killing horizon is $S / 2 \pi$ where $S$ is the black hole entropy. In our case the charge associated with the Killing horizon generating vector fields $\zeta_{\mathcal{H}_{ \pm}}$is $[12,16]$

$\frac{\delta S}{2 \pi}=\frac{\ell}{8 \pi G} \int\left(K_{+} \delta L_{+}+K_{-} \delta L_{-}\right)$.

Using the discussions of the previous subsection we learn that

$\frac{\delta S}{2 \pi}=\beta_{+} \delta J_{+}+\beta_{-} \delta J_{-}, \quad \beta_{ \pm}=\frac{1}{2 \mathcal{T}_{ \pm}}$.

Note that $\delta$ in the above denotes a variation in the Floquet indices $\mathcal{T}_{ \pm}$. Here $\beta_{ \pm}$is the inverse temperature associated with the left and right sectors. The above is nothing but the first law for a generic Bañados geometry. One can integrate (3.12) in the parameter space, over the $\mathcal{T}_{ \pm}$parameters, to obtain the Smarr relation for the Bañados geometries,

$\frac{S}{2 \pi}=\frac{\ell}{4 G}\left(\mathcal{T}_{+}+\mathcal{T}_{-}\right)=2\left(\beta_{+} J_{+}+\beta_{-} J_{-}\right)$.

We can write the entropy as a Cardy-type formula, using $(3.9)^{12}$

$S=2 \pi\left(\sqrt{\frac{c\left(J_{+}+J_{+0}\right)}{6}}+\sqrt{\frac{c\left(J_{-}+J_{-0}\right)}{6}}\right), J_{ \pm 0}=\frac{c}{6} \mathcal{T}_{0 \pm}^{2}$.

One may also compute the conserved charge associated with the inner horizon generating a Killing vector field. Straightforward computation, as performed above, leads to

$\frac{S_{\text {inner }}}{2 \pi}=\frac{\ell}{4 G}\left(\mathcal{T}_{+}-\mathcal{T}_{-}\right)$.

This conserved charge may be written in terms of the other two conserved charges $J_{ \pm}$. In particular one may note that

$S \cdot S_{\text {inner }}=\frac{\pi^{2} \ell}{G}\left(J_{+}-J_{-}\right)$.

\section{Virasoro coadjoint orbits and their associated geometries}

It is well known that the Virasoro algebra,

$\left[L_{n}, L_{m}\right]=(n-m) L_{n+m}+\frac{c}{12} n\left(n^{2}-1\right) \delta_{n+m}$,

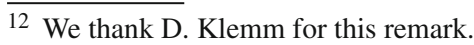


is associated with the algebra of infinitesimal diffeomorphisms on a circle, $\operatorname{diff}\left(S^{1}\right)$. That is,

$x \rightarrow x+\xi(x), \quad \xi(x+2 \pi)=\xi(x)$,

where $\left[\xi(x) \partial_{x}, \rho(x) \partial_{x}\right]$ produces the Witt algebra, the Virasoro algebra without the central term. The Virasoro group, on the other hand, is associated with finite orientationpreserving coordinate transformations on the circle $\operatorname{Diff}\left(S^{1}\right)$, which is generated through

$x \rightarrow h(x), \quad h(x+2 \pi)=h(x)+2 \pi, \quad h^{\prime}>0$.

Being infinite dimensional, representations of the Virasoro group, the corresponding "Virasoro multiplets", are also infinite dimensional. To be precise, we usually deal with "coadjoint orbits" instead of the representations of the Virasoro group. The Virasoro coadjoint orbits are in one-to-one correspondence with Virasoro multiplets [19,3638]. The elements in the Virasoro coadjoint orbits are of course specified by periodic functions on the circle. However, given an arbitrary function $f(x)$ on $S^{1}$ there are elements in $\operatorname{Diff}\left(S^{1}\right)$ under which $f$ does not change. One may then use this fact to distinguish functions (elements) which belong to the same or different orbits. Explicitly, let us recall that from the form of the algebra (4.1) under the transformation (4.2),

$\delta_{\xi} f=\xi^{\prime \prime \prime}-4 f \xi^{\prime}-2 f^{\prime} \xi$,

where the third order derivative appears due to the presence of the central term. ${ }^{13}$ Therefore, all the functions in the same orbit are generated by the solutions to $\delta_{\xi} f=0$, the stabilizer equation [19,36,37]:

$\xi^{\prime \prime \prime}-4 f \xi^{\prime}-2 f^{\prime} \xi=0$.

The orbits associated with function $f(x), \mathcal{O}_{f}$ are then [19, 36-38]

$\mathcal{O}_{f}=\operatorname{Diff}\left(S^{1}\right) / T_{\xi}[f]$,

where $T_{\xi}[f]$ is the subgroup of $\operatorname{Diff}\left(S^{1}\right)$ generated through the periodic solution(s) to the stabilizer equation (4.5); note that only a periodic $\xi(x)$ can be "exponentiated" to give an element of the Virasoro group. So the problem of classification of Virasoro coadjoint orbits reduces to classifying the periodic solutions of the stabilizer equation (4.5). This classification is well established and standard references on the topic are [19,36-38]; however, we will use the method based on the $S L(2, \mathbb{R})$ monodromy discussed in [20] and follow its notations.

\footnotetext{
13 As is implied by the AdS/CFT correspondence the $\delta_{\xi} f$ shows how the energy momentum tensor of a $2 \mathrm{~d}$ CFT, $f$, transforms under conformal transformations.
}

Before moving further, we make the first remarkable correspondence between the analysis of orbits and the Bañados geometries: the stabilizer equation (4.5) is exactly the same equation as appeared in the analysis of Killing vectors of Bañados metrics, and the group $T_{\xi}[f]$ is nothing but the group of global isometries of Bañados metrics with a given $f$.

The rest of the analysis of solutions to (4.5) goes as discussed in the previous section, through the Hill equation,

$\psi^{\prime \prime}-f(x) \psi=0, \quad f(x+2 \pi)=f(x)$,

where $x \in[0,2 \pi]$ parametrizes a circle of unit radius. It is straightforward to check that upon the coordinate transformation in the Virasoro group (4.3), the pair $(\psi(x), f(x))$ in (4.7) transform to $(\tilde{\psi}(x), \tilde{f}(x))$ where [20]

$$
\begin{aligned}
f(x) & \rightarrow \tilde{f}(x)=h^{\prime 2} f(h(x))-S(h ; x), \\
\psi & \rightarrow \tilde{\psi}(x)=\frac{1}{\sqrt{h^{\prime}}} \psi(h(x)),
\end{aligned}
$$

where $S(h ; x)$ is the Schwartz derivative

$S(h ; x)=\frac{h^{\prime \prime \prime}}{2 h^{\prime}}-\frac{3 h^{\prime \prime 2}}{4 h^{\prime 2}}$.

Each coadjoint orbit will hence be specified by a "representative character $f(x)$," the $\psi$ and the corresponding "conformal descendants" [constructed through (4.8)]. The "little group" $T_{\xi}[f]$ by which we mod out the $\operatorname{Diff}\left(S^{1}\right)$, is generically generated by $\psi_{1} \psi_{2}$, where $\psi_{i}$ are the two linearly independent solutions to Hill's equation (4.7) in the Floquet form (2.11). However, in the special case of $f=-n^{2} / 4, n \in \mathbb{Z}$ Hill's equation has three periodic solutions (with $2 \pi / n$ periodicity). In these cases $T_{\xi}[f]$ is an $n$ fold cover of $P L S(2, R), P L S^{(n)}(2, R)[20] . .^{14}$

As reviewed in [20], one can recognize two general classes of such coadjoint orbits: those with a constant representative and the other with $x$-dependent representative.

\subsection{Constant representative coadjoint orbits}

There are four classes of such orbits:

- Exceptional orbits $E_{n}$, with representative:

$$
f_{n}=-\frac{n^{2}}{4}, \quad \psi_{n}=\sqrt{\frac{2}{n}} \sin \frac{n x}{2}, \sqrt{\frac{2}{n}} \cos \frac{n x}{2}, \quad n \in \mathbb{Z}^{+} .
$$

\footnotetext{
${ }^{14}$ As discussed in the next subsection, the case $f(x)=0$ is also special as $\psi_{1}, \psi_{2}$ do not strictly have the Floquet form.
} 
One may write the $\psi$ 's in another Floquet form:

$\psi_{n}=\sqrt{\frac{1}{i n}} e^{i n x / 2}, \sqrt{\frac{1}{i n}} e^{-i n x / 2}$

- Elliptic orbits $C(v)$, with

$f_{v}=-\frac{v^{2}}{4}, \quad \psi_{v}=\sqrt{\frac{2}{v}} \sin \frac{v x}{2}, \sqrt{\frac{2}{v}} \cos \frac{v x}{2}, \quad v \notin \mathbb{Z}^{+}$,

or in the Floquet form

$\psi_{v}=\sqrt{\frac{1}{i \nu}} e^{i \nu x / 2}, \sqrt{\frac{1}{i \nu}} e^{-i v x / 2}$.

Note that there is no overlap between exceptional and elliptic orbits.

- Zeroth hyperbolic orbits $B_{0}(b)$, with

$f_{b}=b^{2}, \quad \psi_{b}=\sqrt{\frac{1}{2 b}} e^{b x}, \sqrt{\frac{1}{2 b}} e^{-b x}, \quad b \in \mathbb{R}^{+}$.

- Zeroth order parabolic orbit $P_{0}^{+}$,

$$
f=0, \quad \psi=\frac{x}{\sqrt{2 \pi}}, \sqrt{2 \pi}
$$

Some comments as regards constant representative orbits are in order:

1. For all of the above orbits the Floquet index is either a real number (for hyperbolic orbits $B_{0}(b)$ ) or a pure imaginary number for exceptional or elliptic orbits $C_{\nu}$.

2. The exceptional case $E_{n}$ is special as it has two Floquet forms, as written in (4.10) and (4.11). The Floquet index in these two cases is either zero or $i n$.

3. The parabolic case is also special in the sense that the corresponding $\psi$ are not in a strict Floquet form, the $\psi_{1}$ is "quasi-periodic", $\psi_{1}(x+4 \pi)=\psi_{1}(x)+2 \psi_{2}(x)$. The generator of the "periodic" Killing case is $\psi_{2}^{2}$ (instead of $\left.\psi_{1} \psi_{2}\right)$.

4. The $b=0$ hyperbolic orbit overlaps with the $n=0$ exceptional orbit, and both have $f=0$. However, this is still different from the parabolic orbit $P_{0}^{+}$, which again has $f=0$.

5. Note that the above functions are for the "representative" of the orbit. A generic element in the orbit may be constructed from these upon the action (4.8) with (4.3). For a generic element in the orbit, hence, the function $f$ is not a constant.
4.2 Nonconstant representative coadjoint orbits

There are two such orbits, parabolic ones $P_{n}^{ \pm}$and hyperbolic ones $B_{n}(b)$ :

- Parabolic orbits $P_{n}^{ \pm}$, with

$$
\begin{aligned}
f_{n}^{ \pm}= & \frac{n^{2}}{2 H_{n}}-\frac{3 n^{2}\left(1 \pm \frac{1}{2 \pi}\right)}{4 H_{n}^{2}}, \\
\psi_{n}= & \frac{1}{\sqrt{H_{n}}}\left( \pm \frac{x}{2 \pi} \sin \frac{n x}{2}-\frac{2}{n} \cos \frac{n x}{2}\right), \\
& \frac{1}{\sqrt{H_{n}}} \sin \frac{n x}{2}, \quad n \in \mathbb{N},
\end{aligned}
$$

where

$$
H_{n}(x)=1 \pm \frac{1}{2 \pi} \sin ^{2} \frac{n x}{2} .
$$

As one can explicitly see, the $\psi$ 's are not in the standard Floquet form, and as the parabolic orbit $P_{0}^{+}, \psi_{2}$ is periodic and $\psi_{1}$ is quasi-periodic,

$\psi_{1}(x+4 \pi)=\psi_{1}(x) \pm 2 \psi_{2}(x)$.

The generator of "periodic" Killing is $\psi_{2}^{2}$ (instead of $\left.\psi_{1} \psi_{2}\right)$. One may easily observe that $H_{n}(x)=$ $H_{n=1}(n x), f_{n}^{ \pm}(x)=n^{2} f_{n=1}^{ \pm}(n x)$. Moreover, $f_{n}^{ \pm}(x)+$ $n^{2} / 4$ is not a positive definite function.

- Hyperbolic orbits $B_{n}(b)$, with

$$
\begin{aligned}
f_{n, b}= & b^{2}+\frac{b^{2}+4 n^{2}}{2 F}-\frac{3 n^{2}}{4 F^{2}}, \\
\psi_{n, b}= & \frac{e^{b x}}{\sqrt{F(x)}} \sqrt{\frac{2}{n}}\left(\frac{b}{n} \cos \frac{n x}{2}+\sin \frac{n x}{2}\right), \\
& \frac{e^{-b x}}{\sqrt{F(x)}} \sqrt{\frac{2}{n}} \cos \frac{n x}{2}, b \in \mathbb{R}^{+}, n \in \mathbb{N},
\end{aligned}
$$

where

$$
F_{n, b}(x)=\cos ^{2} \frac{n x}{2}+\left(\sin \frac{n x}{2}+\frac{2 b}{n} \cos \frac{n x}{2}\right)^{2} .
$$

One may check that

$$
\begin{gathered}
F_{n, b}(x)=F_{n=1, b / n}(n x), f_{n, b}(x)=n^{2} f_{n=1, b / n}(n x), \\
\psi_{n, b}(x)=\sqrt{\frac{2}{n}} \psi_{n=1, b / n}(n x)
\end{gathered}
$$

Moreover, one may check that $f_{n, b}+n^{2} / 4$ is not a positive definite function.

Some comments and points about the nonconstant representatives are in order: 
1. The $n=0$ hyperbolic and parabolic orbits cannot be obtained from the above nonconstant representative orbits by setting $n=0$.

2. The character function $f_{n}$ of the above orbits is a function with $2 \pi / n$ periodicity.

3. The function $f_{n}$ for nonconstant representatives can become negative. The integral of $f_{n}+1 / 4$, which is often called the "energy" [20], has a negative value, except for the $P_{1}^{-}$orbit. For hyperbolic orbits this "energy" is unbounded from below (as a functions of $b$ ). One may show that this "energy" does not have a definite sign either for the descendants of the representative.

4. As the equations above indicate, the representative element of $n>1$ orbits may be expressed through $n=1$ ones, though with replacing $x$ with $n x$. In particular, for the hyperbolic ones one should also replace the $b$ parameter with $b / n$.

5. A generic element in these orbits can be obtained from the above "representatives" upon the action (4.8) with (4.3). For a generic element of the orbit then the character function $f$ and the $\psi$ 's are just $4 \pi$ periodic (and not $4 \pi / n$ periodic).

6. One may readily see that the hyperbolic orbits $B_{n}(b)$ and the exceptional orbits $E_{n}$ overlap at $b=0$.

7. $n$ in fact determines the number of zeros of $\psi$ functions in the $[0,2 \pi]$ range.

\subsection{Orbit invariant quantities}

As discussed, a Virasoro coadjoint orbit is specified by a representative function and a character, $\psi, f$, and then the elements in the orbit, the descendants, are constructed from this upon the action (4.8). So, each orbit consists of infinitely many (countable though, because $h(x)$ is a periodic function) functions/states. Since we built the orbit from the representative, one would expect that the parameters specifying the representative functions should be readable from any element in the orbit (and not just the representative of the orbit). Explicitly, there should be some "orbit invariant" charges and quantities.

In Sect. 3, we have in fact laid the basic foundation for specifying these charges and quantities: any element in an orbit is specified by two kinds of charges: the Virasoro charges (specified by a combination of Virasoro generators $\mathrm{L}_{n}, \Pi_{\left\{n_{k}\right\}} L_{-n_{k}}$ ) and the $J$ charges, which specify the representative. These two charges commute with each other. Our construction of the $J$ charges in Sect. 3.2 makes it explicit that this charge is an orbit invariant quantity.

Now that we have discussed the orbit classification, we can be more explicit about these charges. In general any orbit is specified by a discrete integer label $n$ and/or a continuous label $b$ (for hyperbolic orbits) and $v$ (for elliptic orbits). By construction, the charge $J$ can only be associated with the continuous label on the orbit. The reason is that the method discussed in [16], which is reviewed and used in Sect. 3.2, is suited for computing charge variations within a given class of solutions with exact symmetry. This means that within a class of given orbits, e.g. the hyperbolic orbit with a given $n$, the orbits may be uniquely specified by the continuous label. This continuous parameter is related to the conserved charge $J$, as given in (3.9). We note that the parameter $\mathcal{T}$, which specifies the charge $J$, is the Floquet index defined as $\exp (4 \pi \mathcal{T})=\psi_{1}(x+4 \pi) / \psi_{1}(x)$. With this definition, and recalling (4.8), one immediately sees that $\mathcal{T}$ is orbit invariant, because

$$
\begin{aligned}
e^{4 \pi \mathcal{T}} & =\frac{\tilde{\psi}(x+4 \pi)}{\tilde{\psi}(x)}=\frac{\psi(h(x+4 \pi)) / \sqrt{h^{\prime}}}{\psi(h(x)) / \sqrt{h^{\prime}}} \\
& =\frac{\psi(x+4 \pi)}{\psi(x)} .
\end{aligned}
$$

The discrete label on the orbits is also an orbit invariant quantity. This index is given by the number of zeros of $\psi_{1}$ or $\psi_{2}$ functions in the $[0,2 \pi]$ range. To see the orbit invariance of this label, we recall (4.8), which states that the $\psi$ functions of any two states in the same orbit are related to the representative element as

$$
\begin{aligned}
\tilde{\psi}(x)= & \frac{1}{\sqrt{h(x)}} \psi(h(x)), \\
& h(x+2 \pi)=h(x)+2 \pi, \quad h^{\prime}>0 .
\end{aligned}
$$

The above clearly shows that the number of zeros of $\tilde{\psi}$ and $\psi$ are the same. We note that the $X^{ \pm}$coordinates defined in (2.24) are also orbit invariant.

Unlike the continuous label, the Floquet index, there are no "Noether-type" conserved charges associated with the discrete label $n$. There are however, topological charges (invariants) related to it.

\subsection{Bañados geometries/Virasoro orbits correspondence}

With the discussions and analyses of earlier sections, we are now ready to match the Virasoro coadjoint orbits on the left and right sectors and the Bañados geometries. A discussion of this was presented in Sect. 4 of [12]; our main addition to that list is the cases involving generic hyperbolic orbits. ${ }^{15}$ In general, depending on the $L_{+}$and $L_{-}$ functions, the Bañados geometry will be in an $\mathcal{O}_{+} \otimes \mathcal{O}_{-}$ orbit, where the $\mathcal{O}_{ \pm}$denote the orbits associated with the left and right sectors. Here we will mainly consider the cases where the left and right sectors are from the same class of orbits. Some of the "mixed cases" have been discussed in Appendix A.3.

\footnotetext{
15 A similar analysis and the geometric picture associated with the BMS group has also been discussed in, e.g., Refs. [39-41].
} 
Note that geometries in the same orbits, the descendants, are related to each other by a specific class of diffeomorphisms generated by a vector field $\chi$; see (2.5). Being diffeomorphic to each other, the geometries in the same orbit share the causal, boundary, and horizon structure, and they are of course described with the same $J_{ \pm}$charges and the same horizon temperatures $\mathcal{T}_{ \pm}$.

\section{Constant representative cases}

- $E_{n_{+}} \otimes E_{n_{-}}$orbits. These are geometries which are descendant of $n_{ \pm}$-fold covers of $\mathrm{AdS}_{3}$ with $P L S^{\left(n_{+}\right)}$ $(2, \mathbb{R}) \times P L S^{\left(n_{-}\right)}(2, \mathbb{R})$ isometry. We note that this is the symmetry (isometry) group of the representative of the orbit and the "descendants" generically have only $U(1)_{+} \times U(1)_{-}$global isometry. Note that, while the representatives have $L_{ \pm}=-n_{ \pm}^{2} / 4$, the geometries in this family do not generically have a constant character function $L_{ \pm}$. Geometries in this orbit are horizon free and their global and boundary structure is like $N$-fold cover of $\mathrm{AdS}_{3}$ (see Appendix A.3 for more details). The global $\mathrm{AdS}_{3}$ is the representative element of the $n_{ \pm}=1$ orbit.

- $C\left(v_{+}\right) \otimes C\left(v_{-}\right)$orbits include geometries which are descendants of particles of given mass and angular momentum on the $\mathrm{N}$-fold covers of $\mathrm{AdS}_{3}$ (see Appendix A.3). These geometries do not have a horizon and are not black holes.

- $B_{0}\left(b_{+}\right) \otimes B_{0}\left(b_{-}\right)$orbits. These geometries include BTZ black holes and their (conformal) descendants. We stress that all the geometries in this class (with a given $b_{ \pm}$) have the same $J_{ \pm}$charges. Unlike the usual lore, as we have discussed the correct charge assignment to these geometries is $J_{ \pm}$with the "energy" in the left and right sectors and not $L_{0}$ and $\bar{L}_{0}$. As reviewed in the appendix, the geometry corresponding to the representative of the orbit has constant, positive $L_{ \pm}$, and it is the usual BTZ geometry. The other geometries descending from this constant $L$ ones, are uniquely specified by their Virasoro charges, "Virasoro hairs", while sharing the causal, boundary, and horizon structure of their BTZ parent geometry. All these geometries have the same horizon area, surface gravity, and horizon angular velocity.

- $P_{0}^{+} \otimes P_{0}^{+}$orbit. The representative of this orbit corresponds to a null-self-dual $\mathrm{AdS}_{3}$ orbifold [42] and the other states in this orbit have $J_{ \pm}=0$. These geometries may be obtained in the near horizon limit of the geometry in the orbit of massless BTZ black holes [43]. The global isometry group in this class is $S L(2, \mathbb{R}) \times S L(2, \mathbb{R})$, where the two $U(1)$ factors in $S L(2, \mathbb{R})$ 's can be noncompact.

- $P_{0}^{+} \otimes B_{0}(b)$ orbits. The representative of these geometries is an $\mathrm{AdS}_{3}$ self-dual orbifold [44]. Geometries in this class of orbits are not black holes (do not have event horizons). Nonetheless, they may be obtained as the near horizon limit of extremal BTZ $[12,45]$. The geometries in this class have four global Killing vectors, forming an $S L(2, \mathbb{R}) \times U(1)$ isometry group. Nonetheless, the $U(1) \in S L(2, \mathbb{R})$ is noncompact [12]. ${ }^{16}$

- $P_{0}^{+} \otimes C(v)$ orbits with $0<v<1$. These orbits show "chiral particles" (those with equal mass and angular momentum) on $\mathrm{AdS}_{3}$ and their descendants.

Geometry of hyperbolic orbits, $B_{n_{+}}\left(b_{+}\right) \otimes B_{n_{-}}\left(b_{-}\right)$As follows from the discussions of Sect. 3, these are geometries which in general have inner and outer horizons and their boundary has some disconnected pieces. As a general, but rough, picture one may consider an $N$-fold cover of $\mathrm{AdS}_{3}$ and perform the same construction of BTZ black holes $[7,8]$ on each patch of this $N$-fold cover, such that all the geometries have the same horizon temperatures $\mathcal{T}_{ \pm}$. The horizon and boundary contain an $N$-fold copy and when one starts from a given patch on the boundary, one can only access a specific patch of the horizon (and the geometry). This is the picture we have for multi-BTZ black holes in Brill's construction [27-29]. In the Euclidean version the boundary is a $2 \mathrm{~d}$ surface with $\left(n_{+}+1\right)\left(n_{-}+1\right)$-handles. Irrespective of from which patch at the boundary we look into the bulk, one would see exactly the same geometry, with the same mass and angular momentum.

As we discussed, one can associate positive, definite charges $J_{ \pm}$to these geometries, while the integral of the character $f_{n, b}$ (which has been called the "energy" in the literature, e.g. see [20]) is not necessarily positive definite. We also note that for these orbits generic $n$ and $n=1$ cases they are related as (4.19). This, together with the relation for the entropy (3.14), may suggest that different patches of the horizon, while at the same temperature, carry an equal portion of the entropy, and that each patch has its own "first law" (3.13).

\section{Concluding remarks and outlook}

In this work we elaborated on the Bañados geometries. This work was a continuation of $[12,21]$. Below we provide a quick summary and a general picture arising from our analysis.

1. Different probes can access different kinds of information from the geometry. The "classical" probes, like

\footnotetext{
16 We comment that geometries in the $E_{1} \otimes B_{0}(b)$ orbits, too, have four global Killing vectors forming an $S L(2, \mathbb{R}) \times U(1)$ isometry group, where the two $U(1)$ 's here are compact. These geometries are not black holes either.
} 
geodesics, have only access to "classical, geometric" information. These geometric pieces of information are "orbit invariant" and include geodesic distances, charges associated with the exact Killing symmetries $J_{ \pm}$, and causal and boundary structures. Classical observers are blind to Virasoro charges, "Virasoro hair".

2. The Virasoro charges are semiclassical ones, they are given by "surface integrals," integrals over codimension two space-like curves (that is, integrals over onedimensional curves in our $3 \mathrm{~d}$ case). This information is not available to local classical probes.

3. There is a one-to-one relation between two copies of Virasoro coadjoint orbits and Bañados geometries, which are specified by two general holomorphic functions. All geometries in the same orbit share the "geometric" information, while they can be distinguished by their "Virasoro hairs". The geometric pieces of information are orbit invariant.

4. It is possible that at the level of the geometry we have extra requirements like the absence of CTCs, which need to be considered.

5. Both the charges associated with exact symmetries $J_{ \pm}$ and the Virasoro hairs are "symplectic charges" $[12,16]$. That is, these charges may be defined by integration over any space-like compact curve in the $3 \mathrm{~d}$ spacetime; this curve does not need to be at the boundary of the space or at its horizon.

6. Our analysis suggests the following general picture: different geometries which are diffeomorphic to each other share the "geometric information". This is what we learn in standard GR courses. However, there could be a measure-zero set of diffeomorphisms producing semiclassically different geometries, and they may be distinguished by their other surface charges, "semiclassical hairs". The states sharing the classical geometric information fall into orbits of the semiclassical symmetry algebra. This symmetry algebra is a symplectic symmetry of the phase space constituted from diffeomorphic but distinguishable, geometries. If the geometry we are dealing with is a black hole, then the geometries which share the geometric information may be viewed as "hairs" on this black hole. This, we hope, provides a handle on the black hole microstate problem. We have established this picture for the $\mathrm{AdS}_{3}$ case. Similar ideas have been worked through for near horizon extremal geometries [46,47]. We think this picture should be more general and applicable to any black hole. This picture which was summarized and sharpened in [25] fits well with the recent ideas and analyses [48-50]. We hope to provide further evidence for this picture in more general settings.

7. Although we worked in a specific gauge, the Bañados coordinate system, we believe the above picture is gauge independent. First explicit steps in this direction were taken in [12], where it was shown that similar results hold in the Gaussian null cordinates (also known as the BMS gauge).

8. At a perhaps more technical level, Bañados geometries form a phase space. Elements in this phase space are classified by the Virasoro coadjoint orbits. One may hence use this picture to perform a quantization of the $\mathrm{AdS}_{3}$ gravity. We hope this viewpoint can shed further light on the question of $\mathrm{AdS}_{3}$ gravity quantization, e.g. see [51-55] and references therein.

Acknowledgments We would like to thank Glenn Barnich, Steve Carlip, Geoffrey Compère, Kamal Hajian, Dietmar Klemm, Hai Lin, Joan Simón, Wei Song, and Jun-Bao Wu for discussions and useful comments on the draft. We also thank Dongsu Bak and Soo-Jong Rey for early collaboration. We especially thank Ali Seraj for the discussions as regards the context of Appendix A.1. The work of M.M. Sh-J. is supported in part by Allameh Tabatabaii Prize Grant of Boniad Melli Nokhbegan of Iran, the SarAmadan grant of Iranian vice presidency in science and technology and the ICTP network project NET-68. M.M. Sh-J. also acknowledges the ICTP Simons fellowship. We thank ICTP for the hospitality while this project completed.

Open Access This article is distributed under the terms of the Creative Commons Attribution 4.0 International License (http://creativecomm ons.org/licenses/by/4.0/), which permits unrestricted use, distribution, and reproduction in any medium, provided you give appropriate credit to the original author(s) and the source, provide a link to the Creative Commons license, and indicate if changes were made. Funded by SCOAP $^{3}$.

\section{Appendix A: More on constant representative orbits and their geometries}

In this appendix we give more detailed discussions and computations on the constant $L_{ \pm}$geometries and the associated orbits. This class includes BTZ black holes and conic spaces (particles on $\mathrm{AdS}_{3}$ ).

\section{A.1: More on geometry of constant $L_{ \pm}$cases}

We discussed in Sect. 4 that there are four classes of solutions to the Hill's equation with a constant representative which are elliptic orbits, exceptional orbits, and the zeroth of parabolic and hyperbolic orbits. This gives totally 10 classes of geometry solutions. Four of them correspond to cases with the left and right sectors in the same orbit and others to the mixed orbits. The number of global Killing vectors can be two, four or six. Two general Killing vectors are $\partial_{+}$and $\partial_{-}$. We assume two linearly independent combinations of these two Killing vectors, denoted by $\zeta_{ \pm}=\partial_{+} \pm k \partial_{-}$(with a real non-zero $k$ ). The norm of these vectors is given by

$$
\begin{aligned}
&\left|\zeta_{ \pm}\right|^{2}= \mp \frac{\left(r^{2} \mp \ell^{2} k L_{-}\right)\left(k r^{2} \mp \ell^{2} L_{+}\right)}{r^{2}}, \\
& \zeta_{+} \cdot \zeta_{-}=\ell^{2}\left(L_{+}-k^{2} L_{-}\right) .
\end{aligned}
$$


Depending on the relative signs of $L_{+}$and $L_{-}$, one can distinguish different possibilities. When $L_{+}$and $L_{-}$have opposite signs, the inner product does not vanish anywhere, while $\left|\zeta_{ \pm}\right|$vanish at $r^{2}= \pm \frac{\ell^{2} L_{+}}{k}, r^{2}= \pm k \ell^{2} L_{-}$. When they have similar relative signs, we may take $k^{2}=\frac{L_{+}}{L_{-}}$, leading to

$\left|\zeta_{ \pm}\right|^{2}=\mp \sqrt{\frac{L_{+}}{L_{-}}} \frac{\left(r^{2} \mp \ell^{2} \sqrt{L_{+} L_{-}}\right)^{2}}{r^{2}}, \quad \zeta_{+} \cdot \zeta_{-}=0$,

therefore $r^{2}= \pm \ell^{2} \sqrt{L_{+} L_{-}}$is potentially the position of the bifurcate horizon.

When the $L_{ \pm}$are constant, we can use the coordinate transformation

$$
\begin{aligned}
& \rho^{2}= \frac{\left(r^{2}+\ell^{2} L_{+}\right)\left(r^{2}+\ell^{2} L_{-}\right)}{r^{2}}, \\
& t=\frac{\ell}{2}\left(x^{+}+x^{-}\right), \quad \varphi=\frac{1}{2}\left(x^{+}-x^{-}\right)
\end{aligned}
$$

and rewrite the metric in the "BTZ form"

$\mathrm{d} s^{2}=-F(\rho) \mathrm{d} t^{2}+\frac{\mathrm{d} \rho^{2}}{F(\rho)}+\rho^{2}\left(\mathrm{~d} \varphi-N^{\varphi} \mathrm{d} t\right)^{2}$,

where

$F(\rho)=\frac{\left(\rho^{2}-\rho_{+}^{2}\right)\left(\rho^{2}-\rho_{-}^{2}\right)}{\ell^{2} \rho^{2}}, \quad N^{\varphi}=\frac{\rho_{+} \rho_{-}}{\ell \rho^{2}}$

and

$\rho_{ \pm}=\ell\left(\sqrt{L_{+}} \pm \sqrt{L_{-}}\right)$.

In this coordinate system, (A.2) takes the form

$\left|\zeta_{ \pm}\right|^{2}=\mp\left(\frac{\rho_{+}+\rho_{-}}{\rho_{+}-\rho_{-}}\right)\left(\rho^{2}-\rho_{ \pm}^{2}\right)^{2}$.

As we see for generic $L_{ \pm}$with arbitrary signs, $\rho_{ \pm}$need not be real-valued, and we hence need to consider the three cases of $L_{ \pm}>0, L_{ \pm}<0$, and $L_{+} L_{-}<0$ separately.

$L_{+}, L_{-}>0$, the BTZ case In this case both $\rho_{ \pm}$are realvalued and denote the horizon radii. As (A.6) shows, $\rho_{+}\left(\rho_{-}\right)$ is the radius of the outer (inner) horizon. The event horizon is generated by $\zeta_{\mathcal{H}}$, where

$\zeta_{\mathcal{H}}=\partial_{t}+\Omega \partial_{\varphi}, \quad \Omega=\frac{\rho_{-}}{\ell \rho_{+}}$.

The surface gravity is given by

$\kappa=\frac{\rho_{+}^{2}-\rho_{-}^{2}}{\ell \rho_{+}}$.

To avoid the CTC we need to limit $\rho^{2}$ to the $\rho^{2}>0$ region. Using (A.3) we can readily find where in the Bañados radial coordinate $r^{2}$ is CTC free. This is depicted in Fig. 2. ${ }^{17}$

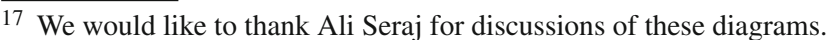

The conserved charges $J_{ \pm}$are related to the mass and angular momentum of the BTZ black hole as in (3.11). In this case the appropriate choice for $\mathcal{T}_{ \pm 0}$ is zero, so that this BTZ has vanishing mass and angular momentum.

$L_{+}, L_{-}<0$, the conic spaces In this case $\rho_{ \pm}^{2}$ are negative and hence there are no Killing horizons $\left(\left|\zeta_{ \pm}\right|=0\right)$ in the region which there are no CTC. These geometries are hence horizonfree and are not black holes. These correspond to particles on $\mathrm{AdS}_{3}$ (the spaces with conical defects) $[5,6]^{18}$. These particles are specified by the mass $M$ and angular momentum $J$,

$\ell M \pm J=J_{ \pm}=\frac{c}{6}\left(\frac{1}{4}+L_{ \pm}\right)$.

The above is nothing but (3.9) with $\mathcal{T}_{ \pm 0}^{2}=-1 / 4$.

To write the metric explicitly, we may introduce $\tilde{\rho}_{ \pm}^{2}=$ $-\rho_{ \pm}^{2}>0$. Then the metric has the same form as (A.4) with

$F(\rho)=\frac{\left(\rho^{2}+\tilde{\rho}_{+}^{2}\right)\left(\rho^{2}+\tilde{\rho}_{-}^{2}\right)}{\ell^{2} \rho^{2}}, \quad N^{\varphi}=-\frac{\tilde{\rho}_{+} \tilde{\rho}_{-}}{\ell \rho^{2}}$.

To avoid the existence of CTC (see Fig. 3), we must restrict ourselves to the $\rho^{2}>0$ region, corresponding to

$r^{2}>0, \quad r^{2} \notin\left(\ell^{2}\left|L_{-}\right|, \ell^{2}\left|L_{+}\right|\right)$.

$L_{+} L_{-}<0$, the mixed case Without loss of generality we may choose $L_{+}>0, L_{-}<0$.

In this case the $\rho_{ \pm}$turn out to be complex conjugate of each others and the metric is given by (A.4) with Fig. 4

$$
\begin{gathered}
F(\rho)=\frac{\left[\rho^{2}-\ell^{2}\left(L_{+}+L_{-}\right)\right]^{2}-4 L_{+} L_{-}}{\ell^{2} \rho^{2}}, \\
N^{\varphi}=\frac{\ell\left(L_{+}+L_{-}\right)}{\rho^{2}} .
\end{gathered}
$$

\section{A.2: Geodesic motion on massless BTZ}

Starting with an $\mathrm{AdS}_{3}$ solution, corresponding to $L_{+}=$ $L_{-}=0$, the metric can be written as

$\mathrm{d} s^{2}=\frac{\ell^{2} \mathrm{~d} \rho^{2}}{\rho^{2}}-\rho^{2} \mathrm{~d} y^{+} \mathrm{d} y^{-}$.

The geodesic equations are given by

$$
\begin{aligned}
& \ddot{\rho}-\frac{\dot{\rho}^{2}}{\rho}+\frac{r^{3} \dot{y}^{+} \dot{y}^{-}}{\ell^{2}}=0, \quad \ddot{y}^{+}+\frac{2 \dot{\rho} \dot{y}^{+}}{\rho}=0, \\
& \ddot{y}^{-}+\frac{2 \dot{\rho} \dot{y}^{-}}{\rho}=0,
\end{aligned}
$$

\footnotetext{
18 See [56] for some discussion of the quantization of conic spaces and the corresponding orbits.
} 

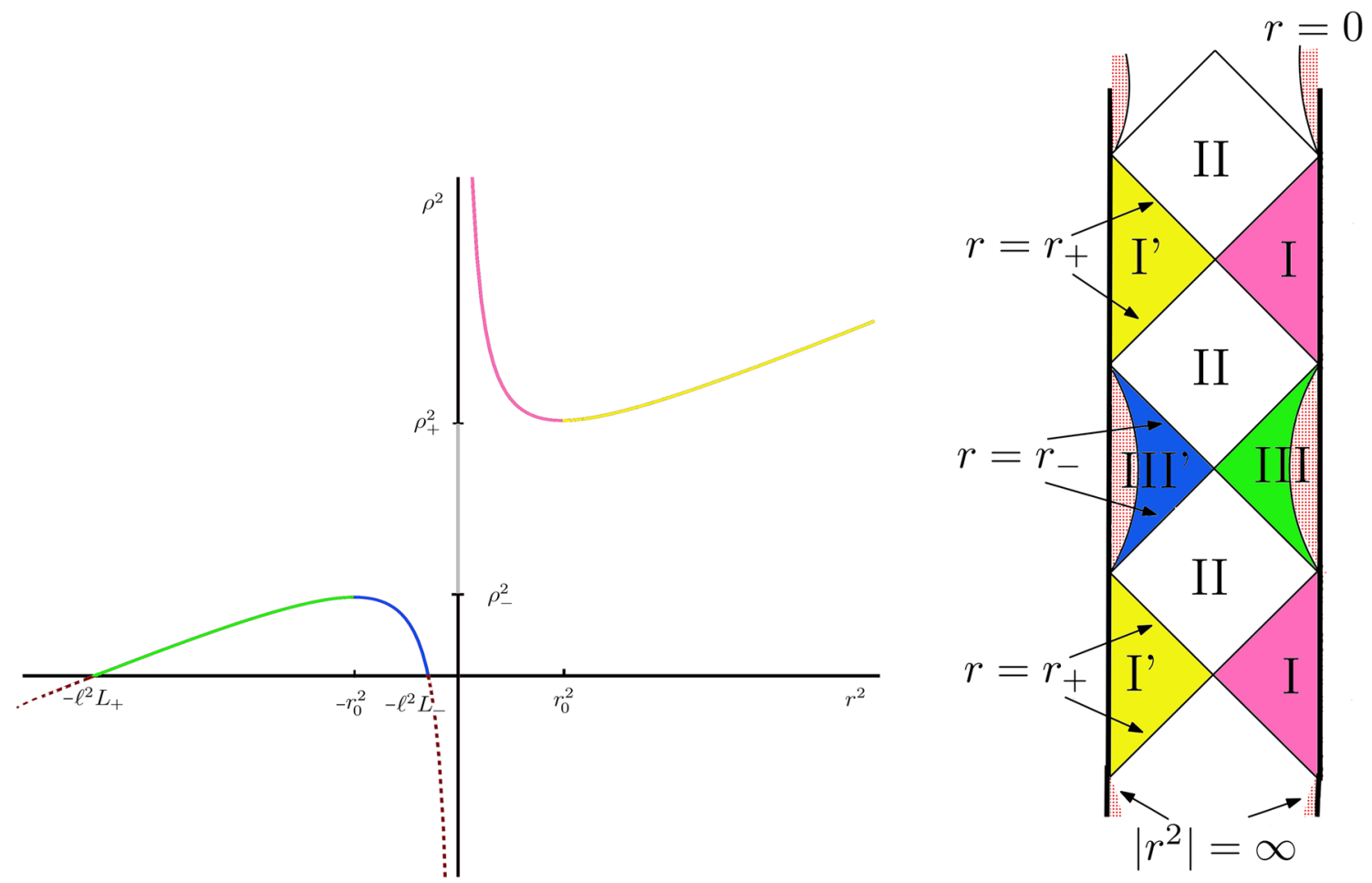

Fig. 2 Left coordinate transformation (A.3) plotted for the BTZ case of $L_{+}>L_{-}>0$. The vertical axis denotes $\rho^{2}$ and the horizontal axis $r^{2}$. The (red) dotted line, where $\rho^{2}<0$, is the location of CTC. Therefore, the CTC-free region in the Bañados coordinate system is $-\ell^{2} L_{+}<r^{2}<-\ell^{2} L_{-}$and $r^{2}>0$. The region on vertical axis in gray color, $\rho_{-}^{2}<\rho^{2}<\rho_{+}^{2}$, is not covered in the Bañados coordinate system, while the other $\rho^{2}>0$ regions are covered twice. The four regions $-\ell^{2} L_{+}<r^{2} \leq-r_{0}^{2},-r_{0}^{2} \leq r^{2}<-\ell^{2} L_{-}, 0<r^{2} \leq r_{0}^{2}$, and $r^{2} \geq r_{0}^{2}$ (with $r_{0}^{2}=\ell^{2} \sqrt{L_{+} L_{-}}$) which are also color-coded in the Left figure, correspond to the four regions, four diamonds, on the Penrose diagram

where a dot denotes the derivative with respect to the proper time $\tau$. The norm of velocity is given by

$\left|\dot{\rho} \partial_{\rho}+\dot{y}^{+} \partial_{+}+\dot{y}^{-} \partial_{-}\right|^{2}=\frac{\ell^{2}}{\rho^{2}} \dot{\rho}^{2}-\rho^{2} \dot{y}^{+} \dot{y}^{-}$,

which is equal to 1 for time-like geodesies and 0 for null geodesies. This equation can be solved to find $\dot{\rho}$ in terms of $\dot{y}^{ \pm}$for the null and time-like geodesies separately; then one may use Eq. (A.13) to solve $\dot{y}^{ \pm}$.

Null geodesies Combining (A.14) and (A.13) for the null geodesy gives us $\ddot{\rho}=0$, which gives $\rho=\frac{2}{\ell} \sqrt{p_{+} p_{-}} \tau$ (after a shift in $\tau$ ). Using this solution we can solve $y^{ \pm}$

$y^{+}-y_{0}^{+}=\frac{\ell^{2}}{2 \tau p_{+}}, \quad y^{-}-y_{0}^{-}=\frac{\ell^{2}}{2 \tau p_{-}}, \quad p_{+} p_{-}, \tau \geq 0$.

Zero angular velocity geodesics correspond to $p_{+}=p_{-}$. In this case
(Right). Right Penrose diagram for the BTZ case of $L_{+}>L_{-}>0$ $[7,8,57]$. Region II (which lies between the inner and outer horizons) is not covered in the Bañados coordinate system. Regions I and I', respectively, correspond to the $0<r^{2} \leq r_{0}^{2}$ and $r^{2} \geq r_{0}^{2}$ regions and regions III and III' to $-\ell^{2} L_{+}<r^{2} \leq-r_{0}^{2},-r_{0}^{2} \leq r^{2}<-\ell^{2} L_{-}$. The shaded regions are where we have CTCs and correspond to the (red) dotted regions in the left figure. We have used the same color-coding in the left and right figures to indicate the range of the $r^{2}$ coordinate. This figure shows how the Bañados and BTZ-coordinate systems are complementary to each other

$y^{+}-y_{0}^{+}=y^{-}-y_{0}^{-}=\frac{\ell}{\rho}$.

Time-like geodesies For the time-like geodesic, by combining (A.14) and (A.13) we get $\ddot{\rho}-\ell^{-2} \rho=0$, which gives $\rho=$ $p_{+} e^{\frac{\tau}{\ell}}-p_{-} e^{-\frac{\tau}{\ell}}$. Using this solution we can solve for $y^{ \pm}$,

$$
\begin{aligned}
y^{+}-y^{0+}= & \frac{\ell p_{m} e^{-\frac{\tau}{\ell}}}{p_{+}\left(p_{+} e^{\frac{\tau}{\ell}}-p_{-} e^{-\frac{\tau}{\ell}}\right)}, \\
& y^{-}-y^{0-}=\frac{\ell e^{-\frac{\tau}{\ell}}}{p_{+} e^{\frac{\tau}{\ell}}-p_{-} e^{-\frac{\tau}{\ell}}} .
\end{aligned}
$$

Null and time-like geodesies of BTZ black holes

Next let us consider a BTZ black hole, i.e., constant nonzero $L_{ \pm}$s. Note that in this case we have only two global Killing vectors $\partial_{ \pm}$. Using the fact that $K . v=$ const. where $K$ is a Killing vector and $v$ is the velocity vector, we get the following equations: 


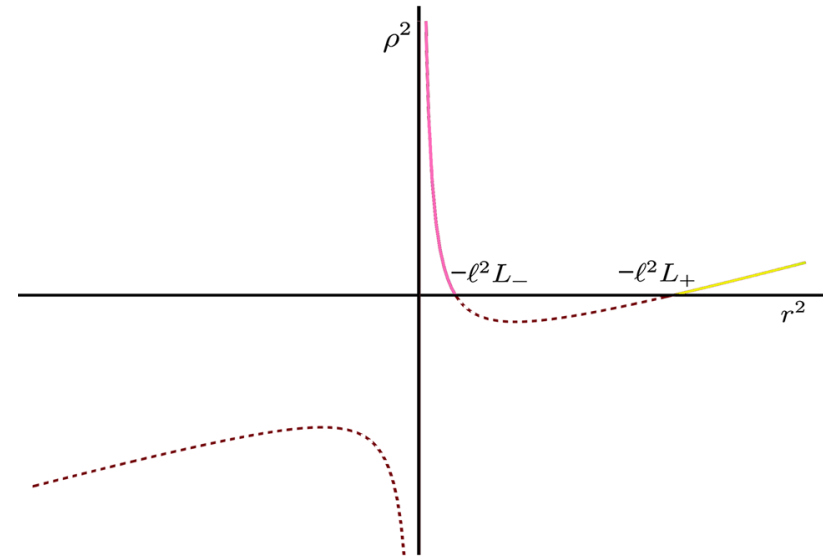

Fig. 3 Coordinate transformation (A.3) plotted for the conic space with $L_{+}<L_{-}<0$. The CTC region, where $\rho^{2}<0$, is denoted by a (red) dotted curve. Extending the coordinate to negative $r^{2}$ gives a CTC. Positive values of $r^{2}$ with $r^{2}>-\ell^{2} L_{+}$gives one cover denoted by the yellow color in the above figure and $r^{2}<-\ell^{2} L_{-}$gives another cover which is denoted by a pink color. The region $-\ell^{2} L_{-}<r^{2}<-\ell^{2} L_{+}$ gives a CTC. Therefore, in the CTC-free range there is no horizon. This is compatible with the fact that conic spaces correspond to particles on $\mathrm{AdS}_{3}$ and not black holes

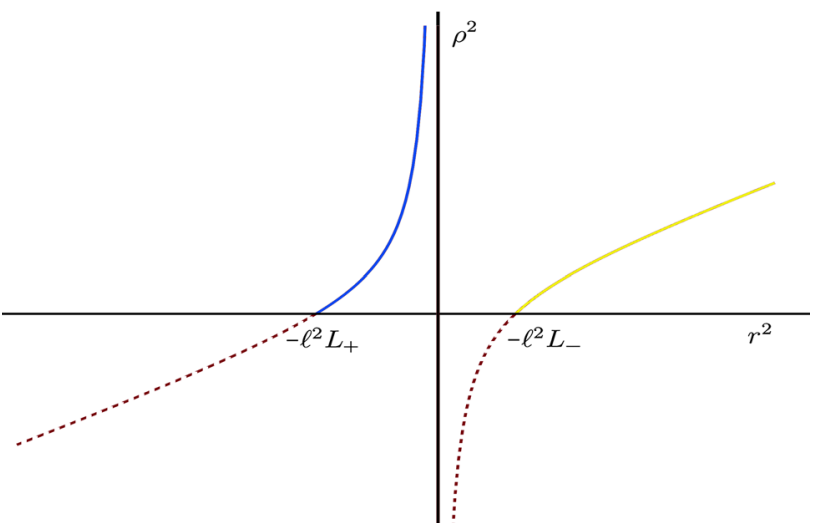

Fig. 4 Coordinate transformation (A.3) plotted for $L_{+}>0$ and $L_{-}<$ 0 . The regions $r^{2}>-\ell^{2} L_{-}$and $-\ell^{2} L_{+}<r^{2}<0$ are the CTC-free regions. The geometry does not have a horizon in this region. The two CTC-free pieces both correspond to the same coordinate range $\rho^{2}>0$ in the BTZ-coordinate system

$$
\begin{gathered}
\ell^{2} L_{+} \dot{x}^{+}-\frac{r^{4}+\ell^{2} L_{+} L_{-}}{2 r^{2}} \dot{x}^{-}=p_{+}, \\
\ell^{2} L_{-} \dot{x}^{-}-\frac{r^{4}+\ell^{2} L_{+} L_{-}}{2 r^{2}} \dot{x}^{+}=p_{-} .
\end{gathered}
$$

These equations give two components of the velocity vector,

$\dot{x}^{ \pm}=-\frac{2 r^{2}\left(r^{4} p_{\mp}+2 \ell^{2} L_{\mp} p_{ \pm} r^{2}+\ell^{4} L_{+} L_{-} p_{\mp}\right)}{\left(r^{4}-\ell^{2} L_{+} L_{-}\right)^{2}}$.

The norm of the velocity vector field is given by

$$
\begin{aligned}
|v|^{2} & =\frac{\ell^{2} \dot{r}^{2}}{r^{2}}-\frac{4 r^{2}\left(p_{+} r^{2}+\ell^{2} L_{+} p_{-}\right)\left(p_{-} r^{2}+\ell^{2} L_{-} p_{+}\right)}{\left(r^{4}-l^{4} L_{+} L_{-}\right)^{2}} \\
& =0,1 . \quad \text { (null, time-like) }
\end{aligned}
$$

Therefore we can read $\dot{r}$ for the null and time-like geodesies.

\section{A.3: More on geometries of constant representative orbits}

As discussed in Sect. 4, Bañados geometries are in one-toone relation with Virasoro coadjoint orbits on the left and right sectors. Here we discuss geometries corresponding to orbits of constant representative in more detail.

Similar constant orbits As we have discussed, the geometric properties, such as the horizon and causal structure, of all the solutions in the same orbit are the same. We will hence only focus on the geometry of the representative element of the orbit.

- $E_{n_{+}} \otimes E_{n_{-}}$orbits In this case the representative element has $L_{ \pm}=-\frac{n_{ \pm}^{2}}{4}$ with integer $n_{ \pm} \cdot n_{+}=n_{-}=1$ corresponds to the global $\mathrm{AdS}_{3}$ geometry. For general $n_{ \pm}$ once again we get six global Killing vectors. However, the periodicities of the Killing vectors are $\frac{2 \pi}{n_{ \pm}}$. If we scale coordinates with the least common multiple of $n_{+}$and $n_{-}$as follows:

$r \rightarrow N r, x^{ \pm} \rightarrow N^{-1} x^{ \pm}, N=\operatorname{lcm}\left(n_{+}, n_{-}\right)$,

the metric turns out to be

$$
\begin{aligned}
\mathrm{d} s^{2}= & \frac{\ell^{2} \mathrm{~d} r^{2}}{r^{2}}-\left(r \mathrm{~d} x^{+}-\frac{\nu_{-}^{2} \ell^{2}}{4 r} \mathrm{~d} x^{-}\right) \\
& \times\left(r \mathrm{~d} x^{-}-\frac{\nu_{+}^{2} \ell^{2}}{4 r} \mathrm{~d} x^{+}\right),
\end{aligned}
$$

where $v_{ \pm}=\frac{n_{ \pm}}{N}$.

When $n_{+}=n_{-}=N, v_{ \pm}=1$ and we get an $n$-fold cover of $\mathrm{AdS}_{3}$. Explicitly if we perform the coordinate transformation (A.3), we arrive at the following metric:

$$
\begin{gathered}
\mathrm{d} s^{2}=-\frac{\rho^{2}+\ell^{2}}{\ell^{2}} \mathrm{~d} t^{2}+\ell^{2} \frac{\mathrm{d} \rho^{2}}{\rho^{2}+\ell^{2}}+\rho^{2} \mathrm{~d} \varphi^{2}, \\
\varphi \in[0,2 \pi N] .
\end{gathered}
$$

For general $n_{+} \neq n_{-}$it is clear that $\nu_{ \pm}<1$, and the metric is like (A.4) with (A.5),

$\rho_{ \pm}^{2}=-\frac{\ell^{2}}{4}\left(v_{+} \pm v_{-}\right)^{2}, \quad \varphi \in[0,2 \pi N]$.

This metric is representing particles on an $N$-fold cover of $\mathrm{AdS}_{3}$; see below.

- $C\left(v_{+}\right) \otimes C\left(v_{-}\right)$orbits When $L_{ \pm}=-\frac{v_{ \pm}^{2}}{4}$ are negative constants with non-integer $v$, the corresponding geometry is a particle in $\mathrm{AdS}_{3}$, if $v_{ \pm}<1$. As discussed in 
Appendix A.1 this geometry does not have an event horizon.

When one or both of $v_{ \pm}$are larger than one, we can rewrite the metric as described for the $E_{n_{+}} \otimes E_{n_{-}}$case above, with

$$
\begin{aligned}
\rho_{ \pm}^{2} & =-\frac{\ell^{2}}{4}\left(\tilde{v}_{+} \pm \tilde{v}_{-}\right)^{2}, \quad \tilde{v}_{ \pm}=\frac{v_{ \pm}}{N}, \\
N & =l c m\left(\left[\nu_{-}\right],\left[v_{+}\right]\right) .
\end{aligned}
$$

As we see, $\tilde{v}_{ \pm}<1$.

- $B_{0}\left(b_{+}\right) \otimes B_{0}\left(b_{-}\right)$orbits When both of $L_{ \pm}$are constant positive $\left(L_{ \pm}=b_{ \pm}^{2}>0\right)$ the corresponding geometry is a BTZ black hole. This solution is discussed in Appendix A.1 in detail.

- $P_{0}^{+} \otimes P_{0}^{+}$orbits The zeroth order of the parabolic orbit has $L=0$. This can also happen in the constant hyperbolic orbits with zero character. The corresponding geometry with $L_{ \pm}=0$ in the parabolic sector is the null self-dual orbifold [42]. The solution is identical to the near horizon limit of a massless BTZ black hole solution [43]. One should note that solutions to the Hill equation in this case do not have the Floquet form, and to construct the global Killing vectors we use only the constant solution. We comment that for the cases with parabolic orbit $P_{0}^{+}$we have the possibilities of having one and three global Killing vectors for each left or right sector. When we have three Killing vectors, the $U(1) \in S L(2, \mathbb{R})$ isometry is not compact.

Mixed constant orbits There are six possible combinations of the left and right sectors with different constant orbits.

- $E_{n} \otimes P_{0}^{+}$orbits The geometry admits four Killing vectors with periodic generators. The geometry associated with the representative of the orbit in this case has the form (A.4) with

$$
F(\rho)=\frac{\left(\rho^{2}+\ell^{2}\right)^{2}}{\ell^{2} \rho^{2}}, \quad N^{\varphi}=\frac{\ell}{\rho^{2}}, \quad \varphi \in[0,2 \pi n] .
$$

- $B_{0}(b) \otimes C(v)$ orbits The corresponding geometry admits only two Killing vectors $\partial_{+}$and $\partial_{-}$. The metric of the representative element takes the general form (A.4) with (A.11).

- $B_{0}(b) \otimes E_{n}$ orbits This geometry has four periodic Killing vectors, three of them with period $\frac{2 \pi}{n}$. The metric for the representative element takes the general form (A.4) with (A.11) with now $L_{+}=b^{2}, L_{-}=-n^{2} / 4$.

- $B_{0}(b) \otimes P_{0}^{+}$orbits In this case the metric of the representative corresponds to the self-dual orbifold of $\mathrm{AdS}_{3}$ [44]. A generic element in this orbit can be obtained as the near horizon limit of the geometry corresponding to $B_{0}(b) \otimes B_{0}(b)$, the extremal BTZ black hole orbit [45].

- $C(v) \otimes E_{n}$ orbits The corresponding geometry admits four Killing vectors; three of them have period $\frac{2 \pi}{n}$. The geometries correspond to chiral particles (those with equal mass and spin) on an $N$-fold $(N=\operatorname{lcm}(n,[v])$ cover of $\mathrm{AdS}_{3}$.

- $C(v) \otimes P_{0}^{+}$orbits The representative geometry corresponds to a chiral particle on $\mathrm{AdS}_{3}$ in a Poincaré patch.

\section{References}

1. E. Witten, (2+1)-Dimensional gravity as an exactly soluble system. Nucl. Phys. B 311, 46 (1988)

2. J.D. Brown, Lower Dimensional Gravity (World Scientific, Singapore, 1988)

3. S. Carlip, Lectures on $(2+1)$ dimensional gravity. J. Korean Phys. Soc. 28, S447 (1995). arXiv:gr-qc/9503024

4. S. Carlip, Quantum gravity in $2+1$ dimensions (Cambridge Univ. Pr, Cambridge, 1998)

5. S. Deser, R. Jackiw, Three-dimensional Einstein gravity: dynamics of flat space. Ann. Phys. 152, 220 (1984)

6. S. Deser, R. Jackiw, Three-dimensional cosmological gravity: dynamics of constant curvature. Ann. Phys. 153, 405 (1984)

7. M. Bañados, C. Teitelboim, J. Zanelli, The Black hole in three-dimensional space-time. Phys. Rev. Lett. 69, 1849 (1992). arXiv:hep-th/9204099

8. M. Bañados, M. Henneaux, C. Teitelboim, J. Zanelli, Geometry of the (2+1) black hole. Phys. Rev. D 48, 1506 (1993). arXiv:gr-qc/9302012

9. J.D. Brown, M. Henneaux, Central charges in the canonical realization of asymptotic symmetries: an example from three-dimensional gravity. Commun. Math. Phys. 104, 207 (1986)

10. K. Skenderis, S.N. Solodukhin, Quantum effective action from the AdS/CFT correspondence. Phys. Lett. B 472, 316 (2000). arXiv:hep-th/9910023

11. M. Bañados, Three-dimensional quantum geometry and black holes. arXiv:hep-th/9901148

12. G. Compère, P.J. Mao, A. Seraj, S. Sheikh-Jabbari, Symplectic and killing symmetries of $\mathrm{AdS}_{3}$ gravity: holographic vs boundary gravitons. JHEP 1601, 080 (2016). arXiv:1511.06079 [hep-th]

13. M. Rooman, P. Spindel, Uniqueness of the asymptotic AdS(3) geometry. Class. Quant. Grav. 18, 2117 (2001). arXiv:gr-qc/0011005

14. A. Garbarz, M. Leston, Classification of boundary gravitons in $\mathrm{AdS}_{3}$ gravity. JHEP 1405, 141 (2014). arXiv:1403.3367 [hep-th]

15. G. Barnich, B. Oblak, Holographic positive energy theorems in three-dimensional gravity. Class. Quant. Grav. 31, 152001 (2014). arXiv: 1403.3835 [hep-th]

16. K. Hajian, M.M. Sheikh-Jabbari, Solution phase space and conserved charges: a general formulation for charges associated with exact symmetries. Phys. Rev. D 93, 044074 (2016). arXiv:1512.05584 [hep-th]

17. J.M. Mitchell, Where are the BTZ Black Hole Degrees of Freedom? Class. Quant. Grav. 32(23), 235009 (2015). arXiv: 1506.04343 [grqc]

18. J.M. Mitchell, Where are the BTZ Black hole degrees of freedom? The rotating case. arXiv:1510.01033 [gr-qc]

19. E. Witten, Coadjoint orbits of the virasoro group. Commun. Math. Phys. 114, 1 (1988) 
20. J. Balog, L. Feher, L. Palla, Coadjoint orbits of the Virasoro algebra and the global Liouville equation. Int. J. Mod. Phys. A 13, 315 (1998). arXiv:hep-th/9703045

21. M.M. Sheikh-Jabbari, H. Yavartanoo, On quantization of $\mathrm{AdS}_{3}$ gravity I: semi-classical analysis. JHEP 1407, 104 (2014). arXiv: 1404.4472 [hep-th]

22. C. Troessaert, Enhanced asymptotic symmetry algebra of $A d S_{3}$. JHEP 1308, 044 (2013). arXiv:1303.3296 [hep-th]

23. C. Fefferman, R. Graham, Conformal invariants. Elie Cartan et les Mathématiques d'aujourd'hui Astérisque, vol. 95 (1985)

24. G. Barnich, A. Gomberoff, H.A. Gonzalez, The flat limit of three dimensional asymptotically anti-de Sitter spacetimes. Phys. Rev. D 86, 024020 (2012). arXiv:1204.3288 [gr-qc]

25. M.M. Sheikh-Jabbari, Residual diffeomorphisms and symplectic hair on Black holes. arXiv:1603.07862 [hep-th]

26. W. Magnus, S. Winkler, Hill's Equation (Dover publications, 2004)

27. D.R. Brill, Multi-black hole geometries in $(2+1)$-dimensional gravity. Phys. Rev. D 53, 4133 (1996). arXiv:gr-qc/9511022

28. D.R. Brill, Black holes and wormholes in $(2+1)$-dimensions. Lect. Notes Phys. 537, 143 (2000). arXiv:gr-qc/9904083

29. S. Aminneborg, I. Bengtsson, D. Brill, S. Holst, P. Peldan, Black holes and wormholes in (2+1)-dimensions. Class. Quant. Grav. 15, 627 (1998). arXiv:gr-qc/9707036

30. K. Skenderis, B.C. van Rees, Holography and wormholes in 2+1 dimensions. Commun. Math. Phys. 301, 583 (2011). arXiv:0912.2090 [hep-th]

31. V. Balasubramanian, P. Hayden, A. Maloney, D. Marolf, S.F. Ross, Multiboundary wormholes and holographic entanglement. Class. Quant. Grav. 31, 185015 (2014). arXiv:1406.2663 [hep-th]

32. J. Lee, R.M. Wald, Local symmetries and constraints. J. Math. Phys. 31, 725 (1990)

33. G. Barnich, F. Brandt, Covariant theory of asymptotic symmetries, conservation laws and central charges. Nucl. Phys. B 633, 3 (2002). arXiv:hep-th/0111246

34. R.M. Wald, Black hole entropy is the Noether charge. Phys. Rev. D 48, 3427 (1993). arXiv:gr-qc/9307038

35. V. Iyer, R.M. Wald, Some properties of Noether charge and a proposal for dynamical black hole entropy. Phys. Rev. D 50, 846 (1994). arXiv:gr-qc/9403028

36. A.A. Kirillov, Funct. Anal. Appl. 15(2), 135 (1981)

37. A.A. Kirillov, in Twistor Geometry and Non-Linear Systems, ed. by H.-D.Doebner, T.D. Palev. Lecture Notes in Mathematics, vol 970 (Springer, Berlin, Heidelberg, 1982), p. 101

38. G. Segal, Unitarity representations of some infinite dimensional groups. Commun. Math. Phys. 80, 301 (1981)

39. G. Barnich, B. Oblak, Notes on the BMS group in three dimensions: I. Induced representations. JHEP 1406, 129 (2014). arXiv:1403.5803 [hep-th]

40. G. Barnich, B. Oblak, Notes on the BMS group in three dimensions: II. Coadjoint representation. JHEP 1503, 033 (2015). arXiv:1502.00010 [hep-th]
41. A. Garbarz, M. Leston, Quantization of $\mathrm{BMS}_{3}$ orbits: a perturbative approach. arXiv:1507.00339 [hep-th]

42. V. Balasubramanian, A. Naqvi, J. Simon, A multiboundary AdS orbifold and DLCQ holography: a universal holographic description of extremal black hole horizons. JHEP 0408, 023 (2004). arXiv:hep-th/0311237

43. J. de Boer, M.M. Sheikh-Jabbari, J. Simon, Near horizon limits of massless BTZ and their CFT duals. Class. Quant. Grav. 28, 175012 (2011). arXiv:1011.1897 [hep-th]

44. O. Coussaert, M. Henneaux, Selfdual solutions of (2+1) Einstein gravity with a negative cosmological constant. in The black hole ed. by C. Teitelboim, pp. 25-39. arXiv:hep-th/9407181

45. V. Balasubramanian, J. de Boer, M.M. Sheikh-Jabbari, J. Simon, What is a chiral 2d CFT? And what does it have to do with extremal black holes? JHEP 1002, 017 (2010). arXiv:0906.3272 [hep-th]

46. G. Compère, K. Hajian, A. Seraj, M.M. Sheikh-Jabbari, Extremal rotating black holes in the near-horizon limit: phase space and symmetry algebra. Phys. Lett. B 749, 443 (2015). arXiv:1503.07861 [hep-th]

47. G. Compère, K. Hajian, A. Seraj, M.M. Sheikh-Jabbari, Wiggling throat of extremal black holes. JHEP 1510, 093 (2015). arXiv: 1506.07181 [hep-th]

48. S.W. Hawking, M.J. Perry, A. Strominger, Soft hair on Black holes arXiv:1601.00921 [hep-th]

49. G. Compre, J. Long, Vacua of the gravitational field. arXiv:1601.04958 [hep-th]

50. G. Compre, J. Long, Classical static final state of collapse with supertranslation memory. arXiv:1602.05197 [gr-qc]

51. S. Carlip, The statistical mechanics of the $(2+1)$-dimensional black hole. Phys. Rev. D 51, 632 (1995). arXiv:gr-qc/9409052

52. S. Carlip, Dynamics of asymptotic diffeomorphisms in $(2+1)$ dimensional gravity. Class. Quant. Grav. 22, 3055 (2005). arXiv:gr-qc/0501033

53. E. Witten, Three-dimensional gravity revisited. arXiv:0706.3359 [hep-th]

54. A. Maloney, E. Witten, Quantum gravity partition functions in three dimensions. JHEP 1002, 029 (2010). arXiv:0712.0155 [hep-th]

55. J. Kim, M. Porrati, On a canonical quantization of 3D anti de sitter pure gravity. JHEP 1510, 096 (2015). arXiv:1508.03638 [hep-th]

56. J. Raeymaekers, Quantization of conical spaces in $3 \mathrm{D}$ gravity. JHEP 1503, 060 (2015). arXiv:1412.0278 [hep-th]

57. F. Loran, M.M. Sheikh-Jabbari, Orientifolded locally $A d S_{3}$ geometries. Class. Quant. Grav. 28, 025013 (2011). arXiv:1008.0462 [hep-th] 